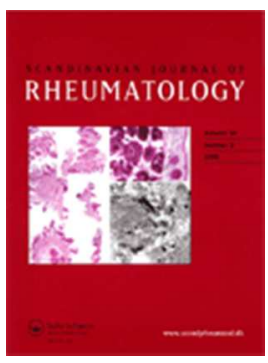

\title{
Systematic review and meta-analysis of the epidemiology of polyautoimmunity in Sjögren's syndrome (secondary Sjögren's syndrome) focusing on autoimmune rheumatic diseases
}

\begin{tabular}{|c|c|}
\hline Journal: & Scandinavian Journal of Rheumatology \\
\hline Manuscript ID & SJR-2016-0514.R2 \\
\hline Manuscript Type: & Article \\
\hline Date Submitted by the Author: & 18-Apr-2017 \\
\hline Complete List of Authors: & $\begin{array}{l}\text { Alani, Hudaifa; Kettering General Hospital NHS Foundation Trust } \\
\text { Henty, Julian; University College London } \\
\text { Thompson, Nicolyn; University College London } \\
\text { Jury, EC; University College London } \\
\text { Ciurtin, Coziana; University College London, Department of Rheumatology }\end{array}$ \\
\hline Keywords: & $\begin{array}{l}\text { secondary Sjogren's syndrome, epidemiology, Meta-analysis, systematic } \\
\text { review, polyautoimunity }\end{array}$ \\
\hline
\end{tabular}




\section{Systematic review and meta-analysis of the epidemiology of}

polyautoimmunity in Sjögren's syndrome (secondary Sjögren's syndrome) focusing on autoimmune rheumatic diseases

\section{Submission type: article}

Short title: Polyautoimmunity in Sjögren's syndrome

Authors:

Hudaifa Alani', Julian R. Henty², Nicolyn L Thompson ${ }^{3}$, Elizabeth Jury ${ }^{3}$

and Coziana Ciurtin ${ }^{3 *}$

${ }^{1}$ Kettering General Hospital, NN16 8UZ, Kettering, United Kingdom

${ }^{2}$ Department of Medical Physics, University College London, London, United Kingdom

${ }^{3}$ Department of Rheumatology, University College London, London, United Kingdom 


\section{*Corresponding author:}

Dr. Coziana Ciurtin, PhD, FRCP, Department of Rheumatology, University College London, 250 Euston Road, London, NW1 2PG, email: c.ciurtin@ucl.ac.uk, phone: +44(0)2034479035, fax: +44(0)20344797268 . 


\section{Abstract}

Objective: The epidemiology of polyautoimmunity in Sjögren's syndrome (secondary Sjögren's syndrome - sSS) is not well-defined and was not investigated before using a systematic approach. We conducted a systematic review of the epidemiology of sSS associated with rheumatoid arthritis (RA), systemic lupus erythematosus (SLE), scleroderma and myositis, assessing the prevalence rates (PRs) and clinical and serological features of sSS.

Methods: A systematic literature search of PubMed and Embase databases (updated to March 2016) was performed to identify all published data on prevalence rate, demographic profile, clinical manifestations, laboratory features and causes of death associated with sSS. The prevalence rates of pSS were summarised with PRs and 95\% CIs.

Results: The literature search identified 1639 citations, out of which 42 fulfilled the inclusion criteria. Only 19 studies had moderate to good quality and were selected for the meta-analysis. According to a random-effects model, the pooled PR for sSS associated with RA was $19.5 \%$ (95\% CI 11.2 to 27.8) and the pooled PR for sSS associated with SLE was 13.96\% (95\% CI 8.88 to 19.04). The female/male ratio of sSS in the RA population was 14.7 (95\% CI 7.09 to 256 ) and 16.82 (95\% CI 1.22 to 32.4 ) in the SLE population.

Conclusion: Prevalence rates of sSS vary widely in different populations. Both meta-analyses conducted in the RA and SLE populations were characterised by a high degree of study heterogeneity. The results of this meta-analysis highlighted the need for better quality population studies.

Keywords: secondary Sjögren's syndrome, polyautoimmunity in Sjögren's syndrome, prevalence, sex ratio, systematic review, meta-analysis. 


\section{Introduction}

Sjögren's syndrome (SS) is a chronic systemic autoimmune disorder, which is associated in the majority of cases with lymphocytic infiltration of exocrine glands and epithelium, feature that is considered the histological hallmark of the disease (1). The T cell mediated attack on salivary and lacrimal glands results in chronic inflammation, which is considered in the majority of cases the leading cause to glandular atrophy and deficient glandular function (2). Although, the temporal relationship between the presence of glandular inflammatory infiltrate and atrophic/fibrotic changes associated with ageing and/or disease progression is difficult to appreciate, the minor salivary gland biopsy have their role in the stratification and prognostication of patients with SS (3).

SS is characterised clinically by symptoms of dry mouth (xerostomia) and dry eyes (xerophthalmia), known as sicca symptoms. SS can progress to affect many organ systems (lung, kidney, gastro-intestinal tract, skin, musculoskeletal, and peripheral and rarely central nervous system), and as a result, other clinical manifestations ranging from mild to more severe disease may occur, including: arthralgias, vasculitis, peripheral neuropathy, renal failure and interstitial lung disease (4). In addition, SS is associated with increased risk of lymphoma (5).

SS can occur either alone as primary SS (pSS) or in association with another well-defined autoimmune condition, such as systemic lupus erythematosus (SLE), rheumatoid arthritis (RA), systemic sclerosis (SSc), or dermatomyositis (DM), in which case it is known as secondary SS (sSS) (6). Although, some researchers prefer to use the term of "polyautoimmunity" associated with SS rather than sSS (7, 8), for the purpose of our systematic review, we used the previously accepted terminology of sSS, which enabled us to identify all the relevant papers. In addition, the term "polyautoimmunity" refers to clusters of autoimmune conditions, which may include or not autoimmune rheumatic diseases (8). Previous papers focused predominantly on prevalence studies and distinct immunologic differences between different sSS subtypes, rather than differences in clinical presentations or controversies regarding the patients' diagnosis. 
The diagnosis of SS cannot be made on a single test or symptom. The variability in presentation of SS has led to a difficulty over the years in establishing universally accepted classification criteria. Therefore, there has been a variation in prevalence estimates in the few epidemiological studies that have been documented, as they used different classification criteria $(1,9)$. Although the clinical features of pSS are relatively wellresearched in large epidemiological studies $(10,11)$, there are very few studies looking at the epidemiology and clinical and serological features of sSS. The authors felt that a systematic review of the epidemiology of sSS was needed in order to establish any significant differences in the presentation of sSS according to the background rheumatic condition of patients that might have impact on their long-term management.

Over the last few decades, diagnostic criteria have varied according to different national and international groups. At present, the most widely accepted and cited criteria are the American-European consensus group classification criteria (AECG), which were published in 2002 as a revision from the original European Study group criteria described in 1996 (2, 12). Interestingly, the presence of anti-Ro/La antibodies has not been included as a mandatory classification criteria for sSS, since it was not shown to be significant in previous analysis (13). New classification criteria for SS were recently developed and validated $(14,15)$. Although, they do not introduce any significant changes, they have been validated in three international patient cohorts, and emphasised the role of expert opinion in diagnosing SS.

\section{Methodology}

We performed a PUBMED and EMBASE search for articles involving humans only, published between 1984 and 2016. The MESH terms used were: secondary Sjögren's, epidemiology of Sjögren's syndrome, secondary Sjögren's and systemic lupus erythematosus, secondary Sjögren's and rheumatoid arthritis, secondary Sjögren's and systemic sclerosis/scleroderma/CREST syndrome, and secondary Sjögren's and myositis. As criteria for study selection, we considered all the studies on the epidemiology, diagnosis and follow-up of patients with sSS. We excluded editorials, commentaries, animal studies, questionnaire studies, case reports, case-series, and studies of treatment (Figure 1). 
We extracted data on prevalence, demographic profile, clinical manifestations, laboratory features, underlying autoimmune diseases and causes of death from the selected articles, where available, and organised them in tables.

Studies were grouped according to the following patient categories: 1). secondary Sjögren's and systemic lupus erythematosus, 2). secondary Sjögren's and rheumatoid arthritis, 3). secondary Sjögren's and scleroderma and 4). secondary Sjögren's and myositis. We present our results data under the following headings: prevalence and demographics of secondary Sjögren's syndrome in different autoimmune diseases, clinical and laboratory features of Sjögren's syndrome in different autoimmune diseases, and morbidity and mortality associated with secondary Sjögren's syndrome. Prevalence rates were calculated using 95\% CI. Pooled prevalence rates and sex ratios were calculated using a random effects model (based on the $\mathrm{Q}$ and $\mathrm{I}^{2}$ tests of heterogeneity among studies).

\section{Results:}

The initial research yielded 1639 articles, which were screened for titles and abstracts, of which 37 were selected for review based on the inclusion and exclusion criteria mentioned above (the detailed process of paper selection is detailed in Figure 1). Following an additional manual search of other relevant articles, we identified 42 full papers and abstracts, which met the inclusion criteria and were analysed further.

We appreciate a risk of reporting bias for the majority of studies as they addressed different populations and used different classification criteria for sSS. The study quality was weighted as poor, moderate and good based on the following criteria: number of patients, the use of established SS classification criteria, data about patient sex, ethnicity and disease duration, inclusion of lip biopsy in the classification criteria (especially as the serology is likely to be positive in lupus patients, irrespective of concomitant SS). The assessment of study quality was reviewed independently by HA and CC. There was an $82 \%$ consensus. The studies in which case the consensus was not reached, were further evaluated by the third author (EJ) and graded based on the assessment made by two of the three authors. The details of study quality assessment were included 
in Table 1. Based on this selection, 9 SLE studies and 11 RA studies assessed as having moderate to good quality were included in the final meta-analysis.

\section{Prevalence and demographics of SS associated with different autoimmune diseases}

Our search identified 40 worldwide studies, which evaluated the prevalence of sSS in patients with RA, SLE, SSc and myositis. The study designs included were retrospective, cross-sectional and prospective (Table 2). The number of patients used in each study ranged from 6 to 2694.

\section{Criteria}

Different criteria were used across the different studies to classify patients as having sSS, including AECG (14), ECC (7), Japanese classification criteria (1), and other criteria (9). Nine studies did not specify the classification criteria they used.

\section{Prevalence}

We found 18 studies which looked at the prevalence of RA-sSS (between 1987-2013) (16-33), 13 for SLE-sSS (1998-2015) (34-46), 6 for SScSSS (1983-2013) (47-52) and 3 for myositis-sSS (2011-2014) (53-55). The prevalence ranged from 3.6\%-55\% for RA-sSS, 5\%-22\% SLE-sSS, $14 \%-60 \%$ SSc-sSS and 10-23\% for myositis-sSS. In the RA-sSS studies three of the highest reported prevalence all came from studies carried out in Greece on Greek patients $(31-39.8 \%)(18,20,23)$.

\section{Gender}

Not all studies reported the gender of the patients, however in the 15 that did, females were predominantly affected (82\%-100\%). Less than half of all studies (17/37) highlighted the ethnicity of the patients included in the study. 
Age

In five studies $(36,39,42,44,45)$ SLE-sSS patients were reported to be significantly older when compared to those with SLE only (48.3 vs. $36.1, p<0.001 ; 41.3$ vs. $35.8, p=0.003 ; 50.8$ vs. $43.6, p=0.01 ; 41$ vs. $35 p=0.03$, and 49.5 vs. $41.4 p<0.001$ ). This was not found to be the case for RA-sSS patients when compared to RA patients as reported by two studies (63.0 vs. 59.2, p=0.33 and 66.36 vs. 62.40, p=NS) (21, 28). There was also no significant difference in age between SSc vs. SSc-sSS patients reported in three studies (56 vs. 54, p=NS; 50.2 vs. 55, p=0.18, and 48.3 vs. $50.5 \mathrm{p}=\mathrm{NS})(50-52)$.

Disease duration

Disease duration was reported in 26/40 studies and varied across all studies ranging from 4 months -12 years for RA-sSS (16, 20-23, 25, 27-29, 32, 33), 3-46 years SLE-sSS (34-37, 39, 41, 42, 44), 7-8 years SSc-sSS (47, 50-52), and 67 months to 20 years for myositis-sSS (53-55).

Only the RA-sSS studies looked into the relationship between disease duration and sSS incidence rate. There were conflicting reports with regard to whether RA disease duration plays any role in the reported occurrence rate of sSS. Two studies found that the cumulative prevalence of sSS did increase with RA duration $(19,29)$. This finding however, was not supported by four other studies $(21,22,25,28)$.

\section{Clinical and laboratory features of sSS associated with different autoimmune diseases:}

We identified 17 studies assessing the clinical and laboratory features of sSS (Tables 3-4). The reported clinical features varied depending on which condition associated with sSS was being looked at. 


\section{SLE-SSS}

Of the seven studies $(36,37,39,42,44-46)$ assessing clinical features of SLE-sSS, the majority reported data on the presence of renal involvement and central nervous system (CNS) involvement. Renal involvement was found to be significantly reduced in SLE-sSS patients across five studies $(36,37,39,44,45)$, while thyroiditis was found to be significantly higher. CNS involvement prevalence, although reduced in SLE-sSS patients, was not significant in any of the three studies. Only one study reported no cases of lymphoma in their cohort of 26 SLE-sSS patients (36), although they reported 8 cases of lymphoma in their pSS comparative group $(\mathrm{N}=86)$.

Regarding serological markers, only anti-Ro/SSA and anti-La/SSB antibodies were significantly raised in SLE-sSS compared to SLE patients in five studies $(36,37,39,42,44)$. Anti-dsDNA was higher in SLE patients and this reached significance in four studies (37, 39, 42, 44). Thrombocytopenia was lower in SLE-sSS patients, although this was only significant in two studies $(36,45)$.

Koskenmies et al. reported that sSS was most commonly observed in patients with subacute cutaneous lupus erythematosus (SCLE) and SLE than in patients with discoid lupus erythematosus ( $16.4 \%$ vs. $22.1 \%$ vs. $2.3 \%$, p<0.001) (43) .

\section{RA-sSS}

Five studies addressed clinical features of RA-sSS patients $(21,22,25,27,28)$. The majority reported data on joint swelling, tender joints and disease activity score assessing 28 joints (DAS 28 score). Half the studies reported that tender joints were significantly higher in RA-sSS patients compared to RA patients $(22,27)$, while the other half reported no significant difference $(21,28)$. Only one reported study looked at other clinical features including lymphadenopathy, thyroiditis, lung, renal and CNS involvement (27).

Laboratory analysis was mainly focused on RF, anti-CCP, CRP and ESR levels. There was inconsistency concerning the reported RF levels. Three studies $(22,25,27)$ reported higher levels of RF in RA-sSS patients, one being significant, while the opposite was found in two other studies, although not significant $(21,28)$. 


\section{$S S c-s S S$}

Four studies $(47,50-52)$ reported data about lung involvement, mainly pulmonary fibrosis (PF) and pulmonary arterial hypertension (PAH). PF occurred significantly less frequently in SSc-sSS patients. Only one study reported lower occurrence of PAH in SSc-sSS, which was significant (52).

The majority of studies looked at anti-topoisomerase 1 antibodies and anti-centromere (ACA) levels. ACA levels were significantly higher in SSc-sSS patients across all three studies (50-52). However, there was discrepancy regarding the prevalence of anti-topoisomerase 1 antibodies.

\section{Myositis-sSS}

In one study, all six patients with both diseases presented with a pattern of muscle weakness typical of IBM (53). Five IBM-sSS patients were treated with prednisolone and methotrexate, four of whom had temporary symptomatic improvement (6-24 months). This was a far greater response in comparison to the IBM only group in whom only $27 \%$ had a transient response to treatment.

Immunogenetics

\section{SLE-SSS}

Immunogenetic analysis was carried out in two studies. In one study there was no significant difference between the SLE and SLE-sSS patients when looking at the HLA alleles (42). However, in another study, the HLA associations distinguished the SLE group from those with SLE-sSS. 
Those with SLE were found to have increased phenotype and allele frequencies for $\mathrm{DRB} 1 * 1501$ ( $\mathrm{p}=0.020$ and $\mathrm{p}=0.015$, respectively) and DQB1*0602 (both $\mathrm{p}<0.001$ ) that was significant (36).

\section{Myositis-sSS}

In the study mentioned above (53), all six patients carried the HLA-DRB1*0301 allele or its equivalent HLA-DR3 serological specificity. They also carried either all or some of the major markers of the $8.1 \mathrm{MHC}$ ancestral haplotype. This allele was also reported to be highly prevalent among the Norwegian patients included in another study (25\%), which found a prevalence of rheumatic disorders of $24 \%$, which is twice as high as previously reported (55).

\section{Morbidity and mortality associated with sSS}

In a few RA studies other aspects of the disease including its effect on quality of life and its involvement in haematological malignancies was studied.

Health status

\section{RA-sSS}

In three of the five studies measuring health status there was no difference in the DAS-28 mean scores in RA-sSS compared to RA patients (21, $25,28)$. In two other studies $(22,27)$ however, a higher DAS-28 score was found in RA-sSS patients, compared to RA patients $(6.44$ vs. 5.96 , $\mathrm{p}=0.02$ and 5.08 vs. $4.20, \mathrm{p}=<0.001$, respectively). 
One study (22) looked at other health status measures including pain visual analogue scale (VAS) scale, fatigue VAS and Modified Health Assessment Questionnaire (M-HAQ), and found that the RA-sSS patients scored significantly higher in all three tests in comparison to RA patients ( 43.1 vs. $32.9, \mathrm{p}=<0.01 ; 49.8$ vs. $39.7, \mathrm{p}=0.03$, and 1.75 vs. $1.55, \mathrm{p}=0.04$, respectively). Another study (28) looked at both M-HAQ and pain VAS tests and reported similar findings in both groups of patients ( 0.84 vs. $0.81, \mathrm{p}=0.7$, and 35.9 vs. 42.4 , p=0.3, respectively).

Haematological malignancy and mortality

\section{SLE-SSS}

Nossent et al. (45) reported a significantly reduced overall mortality in patients with SLE-sSS compared to SLE patients (4\% vs. 13.5\%, $\mathrm{p}=0.01$ ). In two studies in which patients where followed up for three years and 8 years respectively, none of the patients developed lymphoma $(36,45)$.

Martens et al. looked at the survival of sSS patients in a population-based sample in Minnesota, USA between 1976 and 1992. Of the 74 cases $24(33 \%)$ had sSS and 50 (67) had pSS. It found that when compared with the general population, SS patients had increased mortality ( $\mathrm{p}=0.04)$. Furthermore, when studied separately, the mortality was increased in sSS compared to pSS patients $(\mathrm{p}=<0.005)$, with the majority of $\mathrm{sSS}$ patients having associated RA $(\mathrm{p}=0.86)(56)$.

\section{RA-SSS}


With regard to haematological malignancies, studies have reported increased incidence of non-Hodgkin's lymphoma (NHL) in RA-sSS patients. A Finnish study carried out by Kauppi et al. compared the incidence of NHL in 9,469 RA patients and 709 sSS patients. This study found the incidence of NHL to be almost two-fold in patients with RA-sSS (8.7, CI=4.3-1.6) compared to RA patients (4.5, CI=1.5-11) (56).

\section{$S S c-s S S$}

Baldini et al. described a new clinical phenotype of "ACA-positive limited scleroderma/SS overlap syndrome", which in their retrospective study was characterised by a benign SSc clinical course but at a high risk of non-Hodgkin's lymphoma (57).

\section{Meta- analysis of prevalence rates and sex ratios of SSS associated with SLE and RA}

The results of the meta-analysis revealed a pooled prevalence of $13.96 \%$ (95\% CI 8.88 to 19.04$)$ in the SLE population and $19.5 \%$ (95\% CI 11.2 to 27.8) in the RA population. The statistical analysis of the selected studies revealed a high degree of heterogeneity as expected $\left(\mathrm{I}_{2}=99.98\right.$ for the SLE studies and 99.92 for the RA studies; therefore, we used a random effect statistical model for calculating the pooled prevalence). The results of the meta-analysis are presented in Figures 2 and 3.

We also analysed the sex ratio of sSS patients in the SLE and RA populations, which revealed a clear predominance of female patients (four RA studies reported that all their sSS/RA patients were females). The female: male ratios were 16.82 (95\% CI 1.22 to 32.4) for the SLE-sSS patients and 14.7 (95\% CI 7.09 to 256) for the RA-sSS population.

\section{Discussions}


Secondary SS is characterised by a heterogeneity of clinical manifestations, serological markers and symptoms, which are influenced by patients' underlying pathology. Unfortunately, large prospective studies comparing patients with sSS associated with different autoimmune rheumatic diseases are lacking. Previous studies were interested in comparing the clinical and laboratory features of an autoimmune disease alone or associated with sSS (as the majority of the studies included in this systematic review), or aimed to compare the epidemiology of pSS vs. sSS (this was beyond the scope of our systematic analysis). Other papers explored the communality of serological abnormalities and shared clinical picture in distinct autoimmune rheumatic diseases, such as RA and pSS (58), or advocated that the association of SS with multiple autoimmune diseases, is better described as "polyautoimmunity" (7), as discussed in introduction. However, this terminology is not particularly exact in relation to the presence of clinical and serological features of SS in the context of rheumatic conditions (which is the focus of this review), as it also refers to associations of SS with other autoimmune diseases (8).

There is evidence of a great degree of heterogeneity within all these populations; although previous research established that pSS is associated with a different disease phenotype compared to sSS: e.g. pSS patients had a higher frequency of parotid gland enlargement and Ro and La antibody positivity (59), or had significantly higher levels of IL-2 and IL-6 in their saliva (60). An old study also suggested that extraglandular features are more common in pSS compared to sSS (61).

Our study could not address any controversies regarding the accuracy of patients' diagnosis (e.g. many clinicians' might decide based on their expert opinion to diagnose a patient as having RA associated with SS rather than symmetrical polyarthritis in the context of primary SS). In addition, our systematic review does not imply that patients with sSS associated with different ARDs have similar features (e.g. SLE and SS patients might have similar clinical presentation, which is not the case with patients with SS associated with SD or myositis). Even if only moderate-good quality studies were included in the meta-analysis, the studies with poor quality were also detailed in the paper. In addition, our 
systematic review also reported on papers relevant for the article theme, even if not selected by our systematic approach, such as papers referring to patients defined as having overlapping syndromes rather than SSS (57) or which contained no data about sSS prevalence (62).

Our analysis revealed that SSS is more common in women, irrespective of the underlying autoimmune disease. The confidence intervals of sex ratios in the SLE and RA patients with associated sSS reflected again the high heterogeneity of the studies and the inclusion of studies, which reported the presence of sSS exclusively in the female population.

Despite the effort to define the sSS patient population better, clinicians are still unable to answer practical questions regarding the difference in the long term outcome of sSS associated with an autoimmune disease compared to having only an autoimmune disease, or regarding the best way to stratify these patients to enable the choice of the most suitable therapeutic options. It is recognized that sSS is characterised by significant amount of variability in clinical presentation, which is influenced by the concomitant autoimmune disease; however, different studies reported various epidemiological features in the context of similar background autoimmune disease. This variation can be in part explained by the SS classification criteria applied, as well as patient selection criteria, their ethnicity or genetic background, and possible reporting bias (45, 63). Our results show that Raynaud's phenomenon, thyroiditis and Ro, La antibody positivity seemed to be more frequent in SLE-sSS patients compared to SLE group, while renal involvement and presence of dsDNA, and anti Sm antibodies were more common in the latter group. A less clearly defined trend was identified in the case of thrombocytopaenia and lung involvement, while the CNS involvement was reported in only one study as more frequent in the SLE-sSS group.

The number of tender and swollen joints was more commonly reported in the RA compared to RA-sSS group, however, the RA-sSS group had more CNS involvement, Raynaud's phenomenon, thrombocytopaenia and hypergammaglobulinaemia. 
In the scleroderma and myositis groups, the main differences were seen in the positivity of antibodies (the disease characteristic ones being more prevalent in their corresponding group). However, as there was no significant overlap between the clinical features of these autoimmune conditions, and the quality of the studies was poor, no assumptions can be made about the difference in the clinical presentation of SSc-sSS made for patients' clinical phenotype, apart from additional symptoms of dryness, and a possible subset of patients with overlap syndrome with milder disease presentation (57).

Importantly, studies of both SLE and SSc populations hinted at the possibility that these patients have the highest risk of lymphoma of all the autoimmune disease groups $(44,57)$.

Our systematic review included mainly prevalence studies (as the large population studies were lacking) and reported prevalence rather than incidence figures (as SSS is reported in patient groups rather than general population, and the appreciation of the newly diagnosed cases per year necessitates long prospective studies not available in the literature). The quality of the studies included in the analysis was poor to moderate because of the following reasons: significant heterogeneity of patient inclusion criteria (12 studies did not use validated classification criteria and were excluded from the final analysis); variable number of patients included (from 6 to 2694); different proportion of patients classified as having SS based on salivary gland biopsy (14 studies included the biopsy as a classification criteria for all the sSS patients, and 5 studies for a variable proportion of patients); limited geographic areas (only one study evaluated patients from two different countries), and difficulty to extrapolate data to other populations (there were no studies from South America, Africa or Oceania). There was also evidence of significant statistical heterogeneity in our meta-analysis of sSS prevalence in SLE and RA patients, probably due to both, clinical and methodological differences between studies.

The authors identified an unmet need for a consensus regarding the diagnostic/classification criteria for sSS in the context of different underlying autoimmune diseases, especially in the group of SLE/sSS, which is characterised by shared clinical and serological features that make the diagnosis difficult in the absence of a positive salivary gland biopsy. 
In conclusion, this is the first systematic review and meta-analysis of the epidemiology of sSS, which aimed to evaluate the characteristics of this heterogeneous population. Because of the lack of prospective longitudinal data in large population studies, there are still unanswered questions related to the malignancy risk of these patients or their clinical and laboratory features in less common autoimmune diseases.

Acknowledgement: CC was funded by a British Research Council grant (BRC-III/003).

\section{References:}

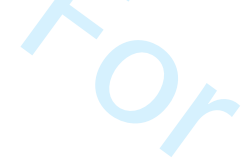

1. $\quad$ Baldini C, Talarico R, Tzioufas AG, Bombardieri S. Classification criteria for Sjogren's syndrome: a critical review. J Autoimmun 2012;39:9-14.

2. Jonsson R, Vogelsang P, Volchenkov R, Espinosa A, Wahren-Herlenius M, Appel S. The complexity of Sjogren's syndrome: novel aspects on pathogenesis. Immunol Lett 2011;141:1-9.

3. Fisher BA, Brown RM, Bowman SJ, Barone F. A review of salivary gland histopathology in primary Sjogren's syndrome with a focus on its potential as a clinical trials biomarker. Ann Rheum Dis 2015;74:1645-50.

4. $\quad$ Patel R, Shahane A. The epidemiology of Sjogren's syndrome. Clin Epidemiol 2014;6:247-55.

5. Johnsen SJ, Brun JG, Goransson LG, Smastuen MC, Johannesen TB, Haldorsen K, et al. Risk of non-Hodgkin's lymphoma in primary Sjogren's syndrome: a population-based study. Arthritis Care Res 2013;65:816-21.

6. Theander E, Jacobsson LT. Relationship of Sjogren's syndrome to other connective tissue and autoimmune disorders. Rheum Dis Clin North Am. 2008;34:935-47, viii-ix.

7. Anaya JM, Rojas-Villarraga A, Mantilla RD, Arcos-Burgos M, Sarmiento-Monroy JC. Polyautoimmunity in Sjogren Syndrome. Rheum Dis Clin North Am 2016;42:457-72.

8. Amador-Patarroyo MJ, Arbelaez JG, Mantilla RD, Rodriguez-Rodriguez A, Cardenas-Roldan J, Pineda-Tamayo R, et al. Sjogren's syndrome at the crossroad of polyautoimmunity. J Autoimmun 2012;39:199-205.

9. Alamanos Y, Tsifetaki N, Voulgari PV, Venetsanopoulou Al, Siozos C, Drosos AA. Epidemiology of primary Sjogren's syndrome in north-west Greece, 1982-2003. Rheumatology (Oxford) 2006;45:187-91.

10. Maldini C, Seror R, Fain O, Dhote R, Amoura Z, De Bandt M, et al. Epidemiology of primary Sjogren's syndrome in a French multiracial/multiethnic area. Arthritis Care Res 2014;66:454-63. 
11. Qin B, Wang J, Yang Z, Yang M, Ma N, Huang F, et al. Epidemiology of primary Sjogren's syndrome: a systematic review and meta-analysis. Ann Rheum Dis 2015;74:1983-9.

12. Vitali C, Bombardieri S, Jonsson R, Moutsopoulos HM, Alexander EL, Carsons SE, et al. Classification criteria for Sjogren's syndrome: a revised version of the European criteria proposed by the American-European Consensus Group. Ann Rheum Dis 2002;61:554-8.

13. Vitali C, Bombardieri S, Moutsopoulos HM, Balestrieri G, Bencivelli W, Bernstein RM, et al. Preliminary criteria for the classification of Sjogren's syndrome. Results of a prospective concerted action supported by the European Community. Arthritis Rheum 1993;36:340-7.

14. Shiboski CH, Shiboski SC, Seror R, Criswell LA, Labetoulle M, Lietman TM, et al. 2016 American College of Rheumatology/European League Against Rheumatism classification criteria for primary Sjogren's syndrome: A consensus and data-driven methodology involving three international patient cohorts. Ann Rheum Dis 2017;76:9-16.

15. Shiboski CH, Shiboski SC, Seror R, Criswell LA, Labetoulle M, Lietman TM, et al. 2016 American College of Rheumatology/European League Against Rheumatism Classification Criteria for Primary Sjogren's Syndrome: A Consensus and Data-Driven Methodology Involving Three International Patient Cohorts. Arthritis Rheum 2017;69:35-45.

16. Turesson C, O'Fallon WM, Crowson CS, Gabriel SE, Matteson EL. Extra-articular disease manifestations in rheumatoid arthritis: incidence trends and risk factors over 46 years. Ann Rheum Dis 2003;62:722-7.

17. Calguneri M, Ureten K, Akif Ozturk M, Onat AM, Ertenli I, Kiraz S, et al. Extra-articular manifestations of rheumatoid arthritis: results of a university hospital of 526 patients in Turkey. Clin Exp Rheumatol 2006;24:305-8.

18. Drosos AA, Lanchbury JS, Panayi GS, Moutsopoulos HM. Rheumatoid arthritis in Greek and British patients. A comparative clinical, radiologic, and serologic study. Arthritis Rheum 1992;35:745-8.

19. Carmona L, Gonzalez-Alvaro I, Balsa A, Angel Belmonte M, Tena X, Sanmarti R. Rheumatoid arthritis in Spain: occurrence of extra-articular manifestations and estimates of disease severity. Ann Rheum Dis 2003;62:897-900.

20. Ioannidis JP, Tarassi K, Papadopoulos IA, Voulgari PV, Boki KA, Papasteriades CA, et al. Shared epitopes and rheumatoid arthritis: disease associations in Greece and meta-analysis of Mediterranean European populations. Semin Arthritis Rheum 2002;31:361-70.

21. Haga HJ, Naderi Y, Moreno AM, Peen E. A study of the prevalence of sicca symptoms and secondary Sjogren's syndrome in patients with rheumatoid arthritis, and its association to disease activity and treatment profile. Int J Rheum Dis 2012;15:284-8.

22. Uhlig T, Kvien TK, Jensen JL, Axell T. Sicca symptoms, saliva and tear production, and disease variables in 636 patients with rheumatoid arthritis. Ann Rheum Dis 1999;58:415-22.

23. Andonopoulos AP, Drosos AA, Skopouli FN, Acritidis NC, Moutsopoulos HM. Secondary Sjogren's syndrome in rheumatoid arthritis. J Rheumatol 1987;14:1098-103.

24. Mattey DL, Gonzalez-Gay MA, Hajeer AH, Dababneh A, Thomson W, Garcia-Porrua C, et al. Association between HLA-DRB1*15 and secondary Sjogren's syndrome in patients with rheumatoid arthritis. J Rheumatol 2000;27:2611-6.

25. Antero DC, Parra AG, Miyazaki FH, Gehlen M, Skare TL. Secondary Sjogren's syndrome and disease activity of rheumatoid arthritis. Rev Assoc Med Bras 2011;57:319-22. 
26. Kosrirukvongs $P$, Ngowyutagon $P$, Pusuwan $P$, Koolvisoot A, Nilganuwong S. Prevalence of dry eye syndrome and Sjogren's syndrome in patients with rheumatoid arthritis. J Med Assoc Thai 2012;95 Suppl 4:S61-9.

27. He J, Ding Y, Feng M, Guo J, Sun X, Zhao J, et al. Characteristics of Sjogren's syndrome in rheumatoid arthritis. Rheumatology (Oxford) 2013;52:1084-9.

28. Abdelghani KB, Mahmoud I, Chatelus E, Sordet C, Gottenberg JE, Sibilia J. Clinical and Serological Features of SjogrenSyndrome in Patients with Rheumatoid Arthritis. Br J Med Med Res 2016;5:1287-93.

29. Young A, Koduri G. Extra-articular manifestations and complications of rheumatoid arthritis. Best Pract Res Clin Rheumatol. 2007;21(5):907-27.

30. Fujita M, Igarashi T, Kurai T, Sakane M, Yoshino S, Takahashi H. Correlation between dry eye and rheumatoid arthritis activity. Am J Ophthalmol. 2005;140:808-13.

31. Martinez Castro E, Olive Marques A, Bonet Llorach M, Carbonell Abello J, Cobo Valeri E, Junca Valdor S. [Rheumatoid arthritis and Sjogren's syndrome. Special reference to the course time of rheumatoid arthritis]. Med Clin (Barc) 1990;94:655-9.

32. Aliko A, Ciancaglini R, Alushi A, Tafaj A. Sicca symptoms, and lacrimal and salivary flow in Albanian patients with rheumatoid arthritis. J Oral Pathol Med 2010;39:651-6.

33. Cimmino MA, Salvarani C, Macchioni P, Montecucco C, Fossaluzza V, Mascia MT, et al. Extra-articular manifestations in 587 Italian patients with rheumatoid arthritis. Rheumatol Int 2000;19:213-7.

34. Prabu A, Marshall T, Gordon C, Plant T, Bawendi A, Heaton S, et al. Use of patient age and anti-Ro/La antibody status to determine the probability of patients with systemic lupus erythematosus and sicca symptoms fulfilling criteria for secondary Sjogren's syndrome. Rheumatology (Oxford) 2003;42:18991.

35. McDonagh JE, Isenberg DA. Development of additional autoimmune diseases in a population of patients with systemic lupus erythematosus. Ann Rheum Dis 2000;59:230-2.

36. Manoussakis MN, Georgopoulou C, Zintzaras E, Spyropoulou M, Stavropoulou A, Skopouli FN, et al. Sjogren's syndrome associated with systemic lupus erythematosus: clinical and laboratory profiles and comparison with primary Sjogren's syndrome. Arthritis Rheum 2004;50:882-91.

37. Gilboe IM, Kvien TK, Uhlig T, Husby G. Sicca symptoms and secondary Sjogren's syndrome in systemic lupus erythematosus: comparison with rheumatoid arthritis and correlation with disease variables. Ann Rheum Dis 2001;60:1103-9.

38. Alonso MD, Martinez-Vazquez F, Riancho-Zarrabeitia L, Diaz de Teran T, Miranda-Filloy JA, Blanco R, et al. Sex differences in patients with systemic lupus erythematosus from Northwest Spain. Rheumatol Int 2014;34:11-24.

39. Pan HF, Ye DQ, Wang Q, Li WX, Zhang N, Li XP, et al. Clinical and laboratory profiles of systemic lupus erythematosus associated with Sjogren syndrome in China: a study of 542 patients. Clin Rheumatol 2008;27:339-43.

40. Lockshin MD, Levine AB, Erkan D. Patients with overlap autoimmune disease differ from those with 'pure' disease. Lupus Sci Med 2015 ;e000084.

41. Aggarwal R, Anaya JM, Koelsch KA, Kurien BT, Scofield RH. Association between Secondary and Primary Sjogren's Syndrome in a Large Collection of Lupus Families. Autoimm Dis 2015;2015:298506.

42. Szanto A, Szodoray P, Kiss E, Kapitany A, Szegedi G, Zeher M. Clinical, serologic, and genetic profiles of patients with associated Sjogren's syndrome and systemic lupus erythematosus. Hum Immunol 2006;67:924-30. 
43. Koskenmies S, Jarvinen TM, Onkamo P, Panelius J, Tuovinen U, Hasan T, et al. Clinical and laboratory characteristics of Finnish lupus erythematosus patients with cutaneous manifestations. Lupus 2008;17:337-47.

44. Baer AN, Maynard JW, Shaikh F, Magder LS, Petri M. Secondary Sjogren's syndrome in systemic lupus erythematosus defines a distinct disease subset. J Rheumatol 2010;37:1143-9.

45. Nossent JC, Swaak AJ. Systemic lupus erythematosus VII: frequency and impact of secondary Sjogren's syndrome. Lupus. 1998;7(4):231-4.

46. Scofield RH, Bruner GR, Harley JB, Namjou B. Autoimmune thyroid disease is associated with a diagnosis of secondary Sjogren's syndrome in familial systemic lupus. Ann Rheum Dis 2007;66:410-3.

47. Osial TA, Jr., Whiteside TL, Buckingham RB, Singh G, Barnes EL, Pierce JM, et al. Clinical and serologic study of Sjogren's syndrome in patients with progressive systemic sclerosis. Arthritis Rheum 1983;26:500-8.

48. Drosos AA, Andonopoulos AP, Costopoulos JS, Stavropoulos ED, Papadimitriou CS, Moutsopoulos HM. Sjogren's syndrome in progressive systemic sclerosis. J Rheum 1988;15:965-8.

49. Drosos AA, Pennec YL, Elisaf M, Lamour A, Acritidis NC, Jouquan JR, et al. Sjogren's syndrome in patients with the CREST variant of progressive systemic scleroderma. J Rheumatol 1991;18:1685-8.

50. Avouac J, Sordet C, Depinay C, Ardizonne M, Vacher-Lavenu MC, Sibilia J, et al. Systemic sclerosis-associated Sjogren's syndrome and relationship to the limited cutaneous subtype: results of a prospective study of sicca syndrome in 133 consecutive patients. Arthritis Rheum 2006;54:2243-9.

51. Salliot C, Mouthon L, Ardizzone M, Sibilia J, Guillevin L, Gottenberg JE, et al. Sjogren's syndrome is associated with and not secondary to systemic sclerosis. Rheum 2007;46:321-6.

52. Kobak S, Oksel F, Aksu K, Kabasakal Y. The frequency of sicca symptoms and Sjogren's syndrome in patients with systemic sclerosis. Int J Rheum Dis 2013;16:88-92.

53. Rojana-udomsart A, Needham M, Luo YB, Fabian V, Walters S, Zilko PJ, et al. The association of sporadic inclusion body myositis and Sjogren's syndrome in carriers of HLA-DR3 and the 8.1 MHC ancestral haplotype. Clin Neurol Neurosurg 2011;113:559-63.

54. Vancsa A, Gergely L, Ponyi A, Lakos G, Nemeth J, Szodoray P, et al. Myositis-specific and myositis-associated antibodies in overlap myositis in comparison to primary dermatopolymyositis: Relevance for clinical classification: retrospective study of 169 patients. Joint Bone Spine 2010;77:125-30.

55. Dobloug GC, Antal EA, Sveberg L, Garen T, Bitter H, Stjarne J, et al. High prevalence of inclusion body myositis in Norway; a population-based clinical epidemiology study. Eur J Neurol. 2015;22:672-e41.

56. Kauppi M, Pukkala E, Isomaki H. Elevated incidence of hematologic malignancies in patients with Sjogren's syndrome compared with patients with rheumatoid arthritis (Finland). Cancer Causes Control 1997;8:201-4.

57. Baldini C, Mosca M, Della Rossa A, Pepe P, Notarstefano C, Ferro F, et al. Overlap of ACA-positive systemic sclerosis and Sjogren's syndrome: a distinct clinical entity with mild organ involvement but at high risk of lymphoma. Clin Exp Rheum 2013;31:272-80.

58. Koszarny A, Majdan M, Dryglewska M, Tabarkiewicz J. Prevalence of selected organ-specific autoantibodies in rheumatoid arthritis and primary Sjogren's syndrome patients. Reumatologia 2015;53:61-8.

59. Hernandez-Molina G, Avila-Casado C, Cardenas-Velazquez F, Hernandez-Hernandez C, Calderillo ML, Marroquin V, et al. Similarities and differences between primary and secondary Sjogren's syndrome. J Rheumatol 2010;37:800-8. 
60. Streckfus C, Bigler L, Navazesh M, Al-Hashimi I. Cytokine concentrations in stimulated whole saliva among patients with primary Sjogren's syndrome, secondary Sjogren's syndrome, and patients with primary Sjogren's syndrome receiving varying doses of interferon for symptomatic treatment of the condition: a preliminary study. Clin Oral Invest 2001;5:133-5.

61. Tishler M, Aharon A, Ehrenfeld M, Avni I, Bendet E, Bombardieri S, et al. Sjogren's syndrome in Israel: primary versus secondary disease. Clin Rheum 1994;13:438-41.

62. Brown LE, Frits ML, lannaccone CK, Weinblatt ME, Shadick NA, Liao KP. Clinical characteristics of RA patients with secondary SS and association with joint damage. Rheumatology (Oxford) 2015;54:816-20.

63. Andonopoulos AP, Skopouli FN, Dimou GS, Drosos AA, Moutsopoulos HM. Sjogren's syndrome in systemic lupus erythematosus. J Rheumatol. 1990;17:201-4.

\section{Figure legends:}

Figure 1: Flowchart of study selection.

Figure 2: Meta-analysis of the prevalence of sSS in SLE patients.

Figure 3: Meta-analysis of the prevalence of sSS in RA patients.

Table 1: Studies reporting the disease duration and relationship with the sSS prevalence in RA patients.

\begin{tabular}{ll}
\hline Study & Delation between \\
& prevalence of sSS and RA
\end{tabular}




\section{disease duration}

\begin{tabular}{lll}
\hline Uhlig et al.,1999 & 12.2 years & None \\
Antero et al., 2011 & $10.2+/-7$ years & None \\
Abdelghani, 2014 & 15.6 years & None \\
Haga et al.,2012 & 10.63 years & None \\
Carmona et al.,2003 & - & Prevalence at 10 years $17 \%$, \\
Young et al.,2000 & 10 years & and $25 \%$ after 30 years \\
& & Prevalence at 1 year $4 \%$ and \\
& & $12 \%$ after 10 years \\
\hline
\end{tabular}


Table 2: Studies included in the systematic review

\begin{tabular}{|c|c|c|c|c|c|c|c|c|c|c|}
\hline & $\begin{array}{l}\text { Study } \\
\text { design }\end{array}$ & Country & $\begin{array}{l}\text { Number } \\
\text { of } \\
\text { patients }\end{array}$ & $\begin{array}{l}\text { Criteria } \\
\text { used }\end{array}$ & $\begin{array}{l}\text { Number } \\
\text { of sSS } \\
\text { cases (\%) }\end{array}$ & Ethnicity & $\begin{array}{l}\text { Female/male } \\
\text { (n) }\end{array}$ & $\begin{array}{l}\text { Disease duration } \\
\text { (years/months) }\end{array}$ & $\begin{array}{l}\text { Salivary gland } \\
\text { biopsy }\end{array}$ & Study quality \\
\hline & & & & & \multicolumn{6}{|c|}{ Systemic lupus erythematosus } \\
\hline $\begin{array}{l}\text { Nossent et al, } \\
1998\end{array}$ & $P$ & $\begin{array}{l}\text { Norway/ } \\
\text { Netherlands }\end{array}$ & 138 & ECC & $\begin{array}{l}N=27 \\
(19.6 \%)\end{array}$ & NA & $22 / 5$ & 8 years & $\begin{array}{ll}12 & \text { patients } \\
\text { tested } & \end{array}$ & Moderate \\
\hline $\begin{array}{l}\text { McDonagh et al, } \\
2000\end{array}$ & $\mathrm{R}$ & $\begin{array}{l}\text { UK } \\
\text { (London) }\end{array}$ & 215 & ECC & $\begin{array}{l}N=28 \\
(13 \%)\end{array}$ & NA & NA & 18 years & All tested & Moderate \\
\hline $\begin{array}{l}\text { Gilboe et al, } \\
2001\end{array}$ & $\mathrm{R}$ & $\begin{array}{l}\text { Norway } \\
\text { (Oslo) }\end{array}$ & 81 & ECC & $\begin{array}{l}N=9 \\
(11 \%)\end{array}$ & Caucasian & $9 / 0$ & 8 years & NA & Moderate \\
\hline $\begin{array}{l}\text { Bowman et al, } \\
2003\end{array}$ & CS & $\begin{array}{l}\text { UK } \\
\text { (Birmingham) }\end{array}$ & 96 & AECG & $\begin{array}{l}N=18 \\
(19 \%)\end{array}$ & Caucasian & $18 / 0$ & 10.9 years & NA & Moderate \\
\hline $\begin{array}{l}\text { Manoussakis et } \\
\text { al, } 2004\end{array}$ & $\mathrm{R}$ & $\begin{array}{l}\text { Greece } \\
\text { (Athens) }\end{array}$ & 283 & AECG & $\begin{array}{l}N=26 \\
(9 \%)\end{array}$ & Caucasian & $26 / 0$ & 3.5 years & All tested & Good \\
\hline $\begin{array}{l}\text { Szanto et al, } \\
2006\end{array}$ & $\mathrm{R}$ & $\begin{array}{l}\text { Hungary } \\
\text { (Debrecen) }\end{array}$ & 362 & AECG & $\begin{array}{l}N=56 \\
(15 \%)\end{array}$ & Caucasian & $52 / 4$ & 8.1 years & All tested & Good \\
\hline $\begin{array}{l}\text { Scofield et al, } \\
2007\end{array}$ & $\mathrm{R}$ & $\begin{array}{l}\text { USA } \\
\text { (Oklahoma) }\end{array}$ & 1138 & NA & $\begin{array}{l}N=169 \\
(15 \%)\end{array}$ & NA & NA & NA & NA & Poor \\
\hline
\end{tabular}




\begin{tabular}{|c|c|c|c|c|c|c|c|c|c|c|}
\hline Pan et al, 2008 & $\mathrm{R}$ & China & 542 & AECG & $\begin{array}{l}N=35 \\
(6 \%)\end{array}$ & Asian Chinese & $32 / 3$ & 3.7 years & All tested & Good \\
\hline $\begin{array}{l}\text { Koskenmies et } \\
\text { al, } 2008\end{array}$ & $\mathrm{R}$ & $\begin{array}{l}\text { Finland } \\
\text { (Helsinki) }\end{array}$ & 77 & NA & $\begin{array}{l}N=17 \\
(22 \%)\end{array}$ & NA & NA & NA & NA & Poor \\
\hline $\begin{array}{l}\text { Baer et al, } \\
2010\end{array}$ & CS & $\begin{array}{l}\text { USA } \\
\text { (Marylands) }\end{array}$ & 1790 & AECG & $\begin{array}{l}N=259 \\
(14.5 \%)\end{array}$ & $\begin{array}{l}\text { White }=70.7 \\
\text { African } \\
\text { American }=25.5 \\
\text { Hispanic= } 1.9 \\
\text { Asian= } 1.2\end{array}$ & $253 / 6$ & 19.5 years & All tested & Good \\
\hline Maria et al, 2013 & $\mathrm{CS} / \mathrm{R}$ & $\begin{array}{l}\text { Spain } \\
\text { (Lugo) }\end{array}$ & 150 & NA & $\begin{array}{l}N=27 \\
(18 \%)\end{array}$ & NA & $27 / 0$ & NA & NA & Poor \\
\hline $\begin{array}{l}\text { Lockshin et al, } \\
2015\end{array}$ & $\mathrm{R}$ & USA & 600 & Other & $\begin{array}{l}N=28 \\
(5 \%)\end{array}$ & NA & NA & NA & NA & Poor \\
\hline $\begin{array}{l}\text { Aggarwal et al, } \\
2015\end{array}$ & $\mathrm{~L}$ & USA & 2694 & AECG & $\begin{array}{l}N=548 \\
(20 \%)\end{array}$ & $\begin{array}{l}\text { Mixed (White, } \\
\text { Black, } \\
\text { Hispanic, } \\
\text { Asian and } \\
\text { native) }\end{array}$ & $504 / 44$ & 20 years & NA & Good \\
\hline
\end{tabular}

Rheumatoid arthritis

\begin{tabular}{|l|l|l|l|l|l|l|l|l|l|}
\hline $\begin{array}{l}\text { Andonopoulos et } \\
\text { al, } 1987\end{array}$ & $\mathrm{P}$ & Greece & 111 & Other & $\begin{array}{l}\mathrm{N}=34 \\
(31 \%)\end{array}$ & Greek & NA & $3.3-9.1$ years & All tested \\
\hline $\begin{array}{l}\text { Martinez Castro } \\
\text { et al, } 1990\end{array}$ & CS & Spain & 45 & NA & $\begin{array}{l}\mathrm{N}=24 \\
(55 \%)\end{array}$ & Spanish & NA & NA & All tested \\
\end{tabular}




\begin{tabular}{|c|c|c|c|c|c|c|c|c|c|c|}
\hline $\begin{array}{l}\text { Drosos et al, } \\
1992\end{array}$ & CS & $\begin{array}{l}\text { Greece/British } \\
\text { loannina/London }\end{array}$ & $\begin{array}{l}G=108 \\
B=107\end{array}$ & Other & $\begin{array}{l}\text { GK N=43 } \\
(39.8 \%) \\
\text { BS } N=17 \\
(15.9 \%)\end{array}$ & Greek/British & NA & NA & All tested & Moderate \\
\hline Uhlig et al, 1999 & CS & $\begin{array}{l}\text { Norway } \\
\text { (Oslo) }\end{array}$ & 636 & $\mathrm{ECCN}$ & $\begin{array}{l}N=46 \\
(7 \%)\end{array}$ & NA & NA & 12.2 years & NA & Moderate \\
\hline $\begin{array}{l}\text { Cimmino et al, } \\
2000\end{array}$ & CS & $\begin{array}{l}\text { Italy } \\
\text { (Northern) }\end{array}$ & 587 & Other & $\begin{array}{l}N=103 \\
(17.5 \%)\end{array}$ & Italian & NA & 10 years & NA & Poor \\
\hline $\begin{array}{l}\text { Mattey et al, } \\
2000\end{array}$ & CS & $\begin{array}{l}\text { Spain } \\
\text { (Lugo) }\end{array}$ & 179 & Other & $\begin{array}{l}\mathrm{N}=22 \\
(12.3 \%)\end{array}$ & NA & NA & NA & NA & Poor \\
\hline $\begin{array}{l}\text { Young et al, } \\
2000\end{array}$ & $P$ & UK & 732 & NA & $\begin{array}{l}N=54 \\
(7 \%)\end{array}$ & NA & $46 / 9$ & $4-11$ months & NA & Poor \\
\hline $\begin{array}{l}\text { loannidis et al, } \\
2002\end{array}$ & CS & $\begin{array}{l}\text { Greece } \\
\text { (loannina/ } \\
\text { Athens) }\end{array}$ & 174 & ECC & $\begin{array}{l}N=57 \\
(32.7 \%)\end{array}$ & Greek & NA & 10.5 years & NA & Moderate \\
\hline $\begin{array}{l}\text { Turesson et al, } \\
2003\end{array}$ & $\mathrm{R}$ & $\begin{array}{l}\text { USA } \\
\text { (Minnesota) }\end{array}$ & 609 & Other & $\begin{array}{l}\mathrm{N}=58 \\
(9.5 \%)\end{array}$ & NA & NA & 46 years & NA & Poor \\
\hline $\begin{array}{l}\text { Carmona et al, } \\
2003\end{array}$ & CS & Spain & 788 & ECC & $\begin{array}{l}N=134 \\
(17 \%)\end{array}$ & Spanish & NA & NA & NA & Moderate \\
\hline Fujita et al, 2005 & $P$ & $\begin{array}{l}\text { Japan } \\
\text { (Tokyo) }\end{array}$ & 72 & Japanese & $\begin{array}{l}N=7 \\
(10 \%)\end{array}$ & Japanese & $7 / 0$ & NA & NA & Poor \\
\hline $\begin{array}{l}\text { Calgüneri et al, } \\
2006\end{array}$ & $\mathrm{R}$ & $\begin{array}{l}\text { Turkey } \\
\text { (Ankara) }\end{array}$ & 526 & NA & $\begin{array}{l}N=28 \\
(5.3 \%)\end{array}$ & NA & NA & NA & NA & Poor \\
\hline $\begin{array}{l}\text { Antero et al, } \\
2011\end{array}$ & CS & $\begin{array}{l}\text { Brazil } \\
\text { (Curitiba) }\end{array}$ & 82 & AECG & $\begin{array}{l}N=20 \\
(24.3 \%)\end{array}$ & NA & $18 / 2$ & 10.2 years & All tested & Good \\
\hline
\end{tabular}




\begin{tabular}{|c|c|c|c|c|c|c|c|c|c|c|}
\hline $\begin{array}{l}\text { Kosrirukvongs et } \\
\text { al, } 2012\end{array}$ & CS & $\begin{array}{l}\text { Thailand } \\
\text { (Siriraj) }\end{array}$ & 61 & Other & $\begin{array}{l}N=14 \\
(22.2 \%)\end{array}$ & NA & NA & NA & NA & Poor \\
\hline Haga et al, 2012 & CS & $\begin{array}{l}\text { Denmark } \\
\text { (Esbjerg) }\end{array}$ & 307 & AECG & $\begin{array}{l}N=11 \\
(3.6 \%)\end{array}$ & NA & NA & 10.6 years & NA & Moderate \\
\hline Aliko et al, 2010 & CS & $\begin{array}{l}\text { Albania } \\
\text { (Tirana) }\end{array}$ & 88 & ECC & $\begin{array}{l}N=13 \\
(14.8 \%)\end{array}$ & Albanian & NA & 9.5 years & NA & Moderate \\
\hline He J et al, 2013 & $\mathrm{R}$ & $\begin{array}{l}\text { China } \\
\text { (Beijing) }\end{array}$ & 509 & AECG & $\begin{array}{l}\mathrm{N}=74 \\
(14.5 \%)\end{array}$ & Chinese & $64 / 10$ & 15.10 months & $\begin{array}{ll}16 & \text { patients } \\
\text { tested } & \end{array}$ & Good \\
\hline $\begin{array}{l}\text { Abdelghani, } \\
2014\end{array}$ & CS & $\begin{array}{l}\text { France } \\
\text { (Strasbourg) }\end{array}$ & 76 & AECG & $\begin{array}{l}N=11 \\
(14 \%)\end{array}$ & French & NA & 15.6 years & 9 patients tested & Moderate \\
\hline $\begin{array}{l}\text { Brown et al., } \\
2015\end{array}$ & CS & $\begin{array}{l}\text { USA } \\
\text { (Boston) }\end{array}$ & 829 & AECG & $\begin{array}{l}N=85 \\
(10.3 \%)\end{array}$ & White $=89.4$ & $76 / 9$ & 16.9 years & NA & Good \\
\hline \multicolumn{11}{|c|}{ Systemic sclerosis } \\
\hline Osial et al, 1983 & CS & $\begin{array}{l}\text { USA } \\
\text { (Pennsylvania) }\end{array}$ & 58 & Other & $\begin{array}{l}N=17 \\
(29 \%)\end{array}$ & NA & $16 / 1$ & 7.3 years & All tested & Good \\
\hline $\begin{array}{l}\text { Andonopoulos et } \\
\text { al, } 1988\end{array}$ & $P$ & Greece & 44 & Other & $\begin{array}{l}N=9 \\
(20.5 \%)\end{array}$ & NA & NA & NA & All tested & Poor \\
\hline $\begin{array}{l}\text { Drosos et al, } \\
1991\end{array}$ & CS & Greece & 23 & NA & $\begin{array}{l}N=14 \\
(60 \%)\end{array}$ & NA & NA & NA & All tested & Poor \\
\hline $\begin{array}{l}\text { Avouac et al, } \\
2006\end{array}$ & $P$ & $\begin{array}{l}\text { France } \\
\text { (Paris) }\end{array}$ & 133 & AECG & $\begin{array}{l}N=19 \\
(14 \%)\end{array}$ & NA & $16 / 3$ & 7 years & $\begin{array}{ll}91 & \text { patients } \\
\text { tested } & \end{array}$ & Good \\
\hline
\end{tabular}




\begin{tabular}{|c|c|c|c|c|c|c|c|c|c|c|}
\hline Salliot et al, 2006 & $\mathrm{R}$ & $\begin{array}{l}\text { France } \\
\text { (Paris) }\end{array}$ & 121 & AECG & $\begin{array}{l}N=27 \\
(22 \%)\end{array}$ & NA & $24 / 3$ & 7.3 years & All tested & Good \\
\hline Kobak et al, 2013 & CS & $\begin{array}{l}\text { Turkey } \\
\text { (Izmir) }\end{array}$ & 118 & AECG & $\begin{array}{l}N=40 \\
(33.9 \%)\end{array}$ & NA & $38 / 2$ & 8.2 years & $\begin{array}{ll}74 & \text { patients } \\
\text { tested } & \end{array}$ & Good \\
\hline $\begin{array}{l}\text { Baldini et al., } \\
2013\end{array}$ & $\mathrm{R}$ & Italy (Pisa) & $\begin{array}{l}\text { 209- } \\
\text { systemic } \\
\text { sclerosis } \\
402 \\
\text { pSS }\end{array}$ & $\begin{array}{l}\text { Le Roy } \\
\text { AECG }\end{array}$ & $\mathrm{N}=41$ & NA & NA & NA & NA & Moderate \\
\hline \multicolumn{11}{|c|}{ Myositis } \\
\hline $\begin{array}{l}\text { Rojana- } \\
\text { Udomsart et al, } \\
2011\end{array}$ & CS & Australia & 6 & AECG & $\begin{array}{l}N=6 \\
(12 \%)\end{array}$ & NA & $5 / 1$ & 20 years & NA & Poor \\
\hline $\begin{array}{l}\text { Vancsa et al, } \\
2010\end{array}$ & $\mathrm{CS} / \mathrm{R}$ & Hungary & 169 & NA & $\begin{array}{l}N=9 \\
(23 \%)\end{array}$ & NA & NA & 6 years & NA & Poor \\
\hline $\begin{array}{l}\text { Dobloug et al, } \\
2014\end{array}$ & CS & Norway & 100 & NA & $\begin{array}{l}N=10 \\
(10 \%)\end{array}$ & NA & NA & 5.5 years & NA & Poor \\
\hline
\end{tabular}

Legend: CS - cross-sectional study; NA - information not available; P - prospective study; R - retrospective study. 
Table 3 : Clinical features of patients with sSS

\begin{tabular}{|c|c|c|c|c|c|c|c|c|c|c|c|c|}
\hline Disease/Ref & $\begin{array}{l}\text { Arthritis } \\
(\% \\
\text { patients) }\end{array}$ & $\begin{array}{l}\text { Swollen } \\
\text { joints } \\
\text { (\% } \\
\text { patients) }\end{array}$ & $\begin{array}{l}\text { Tender } \\
\text { joints } \\
\text { (\% } \\
\text { patients) }\end{array}$ & $\begin{array}{l}\text { DAS } 28 \\
\text { score }\end{array}$ & $\begin{array}{l}\text { Raynaud } \\
\text { s } \\
\text { (\% } \\
\text { patients) }\end{array}$ & $\begin{array}{l}\text { Photo } \\
\text { sensitivity } \\
(\% \\
\text { patients) }\end{array}$ & $\begin{array}{l}\text { Lymph } \\
\text { adenopathy } \\
\text { (\% patients) }\end{array}$ & $\begin{array}{l}\text { Thyroiditis } \\
\text { (\% } \\
\text { patients) }\end{array}$ & $\begin{array}{l}\text { Lung } \\
\text { involvement } \\
\text { (\% } \\
\text { patients) }\end{array}$ & $\begin{array}{l}\text { Renal } \\
\text { involvement } \\
\text { (\% } \\
\text { patients) }\end{array}$ & $\begin{array}{l}\text { Nervous } \\
\text { system } \\
\text { involvement } \\
\text { (\% patients) }\end{array}$ & $\begin{array}{l}\text { Lymphoma } \\
\text { (\% patients) }\end{array}$ \\
\hline \multicolumn{13}{|c|}{ SLE vs. SLE-sSS patients } \\
\hline $\begin{array}{l}\text { Nossent et al, } \\
1998\end{array}$ & $\begin{array}{l}88 \text { vs. } 92 \\
(p=N S)\end{array}$ & & & & & & & & & $\begin{array}{l}38 \text { vs. } 19 \\
(p=\mathbf{0 . 0 4})\end{array}$ & $\begin{array}{l}\text { NPSLE } \\
18 \text { vs. } 19 \\
(p=N S)\end{array}$ & $0 \%$ \\
\hline $\begin{array}{l}\text { Gilboe et al, } \\
2001\end{array}$ & & & & & & & & & & $\begin{array}{l}19 \text { vs. } 0 \\
(\mathbf{p}<\mathbf{0 . 0 5})\end{array}$ & & \\
\hline $\begin{array}{l}\text { Manoussakis et } \\
\text { al, } 2004\end{array}$ & $\begin{array}{l}51.3 \text { vs. } \\
76.9 \\
(p=0.27)\end{array}$ & & & & $\begin{array}{l}43.4 \text { vs. } \\
80.8 \\
(\mathbf{p}<\mathbf{0 . 0 0 1})\end{array}$ & & $\begin{array}{c}46.1 \text { Vs. } 19.2 \\
(\mathbf{p}=\mathbf{0 . 0 0 4})\end{array}$ & & $\begin{array}{l}11.8 \text { vs. } 11.5 \\
(p=0.891)\end{array}$ & $\begin{array}{l}55.3 \text { vs. } 11.5 \\
(p=\mathbf{0 . 0 0 5})\end{array}$ & $\begin{array}{l}19.7 \text { vs. } 11.5 \\
(p=0.55)\end{array}$ & $0 \%$ \\
\hline $\begin{array}{l}\text { Szanto et al, } \\
2006\end{array}$ & & & & & $\begin{array}{l}28 \text { vs. } 35.7 \\
(p=0.396)\end{array}$ & & & $\begin{array}{l}6 \text { vs. } 21.4 \\
(p=0.023)\end{array}$ & $\begin{array}{l}24 \text { vs. } 28.5 \\
(p=0.59)\end{array}$ & $\begin{array}{l}66 \text { vs. } 57.1 \\
(p=0.312)\end{array}$ & $\begin{array}{l}36 \text { vs. } 25 \\
(p=0.21)\end{array}$ & \\
\hline $\begin{array}{l}\text { Scofield et al, } \\
2007\end{array}$ & & & & & & & & $\begin{array}{l}12.7 \text { vs. } 29.6 \\
(\mathbf{p}<\mathbf{0 . 0 0 0})\end{array}$ & & & & \\
\hline Pan et al, 2008 & & & $\begin{array}{l}60.9 \text { vs. } \\
77.1 \\
(p=0.056)\end{array}$ & & & $\begin{array}{l}14.2 \text { vs. } \\
8.6 \\
(p=0.45)\end{array}$ & & & & $\begin{array}{l}66.7 \text { vs. } 48.6 \\
(\mathbf{p}=\mathbf{0 . 0 3})\end{array}$ & $\begin{array}{l}9.5 \text { vs. } 2.9 \\
(p=0.35)\end{array}$ & \\
\hline $\begin{array}{l}\text { Baer et al, } \\
2010\end{array}$ & $\begin{array}{l}73.2 \text { vs. } \\
81.3 \\
(p=0.006)\end{array}$ & & & & $\begin{array}{l}49.5 \text { vs. } 66 \\
(\mathbf{p}<\mathbf{0 . 0 0 1})\end{array}$ & $\begin{array}{l}52.9 \text { vs. } \\
68.3 \\
(\mathbf{p}=\mathbf{0 . 0 0 1})\end{array}$ & & & & $\begin{array}{l}\begin{array}{l}\text { Proteinuria } \\
43.1 \text { vs. } 29.0 \\
(\mathbf{p}<\mathbf{0 . 0 0 1 )}\end{array} \\
\text { Haematuria } \\
30.9 \text { vs. } 22.8 \\
(\mathbf{p}=\mathbf{0 . 0 0 8}) \\
\text { Nephrotic } \\
\text { syndrome } \\
20 \text { vs. } 8.9 \\
(\mathbf{p}<\mathbf{0 . 0 0 1 )}\end{array}$ & $\begin{array}{l}\text { Psychosis } \\
3 \text { vs. } 6.6 \\
(\mathbf{p}=\mathbf{0 . 0 0 5}) \\
\text { Seizures } \\
9.3 \text { vs. } 11.6 \\
(p=0.25)\end{array}$ & \\
\hline
\end{tabular}




\begin{tabular}{|c|c|c|c|c|c|c|c|c|c|c|}
\hline \multicolumn{11}{|c|}{ RA vs. RA-sSS patients } \\
\hline $\begin{array}{l}\text { Uhlig et al, } \\
1999\end{array}$ & 100 & $\begin{array}{l}7.0 \text { vs. } \\
8.5 \\
(p=0.17)\end{array}$ & $\begin{array}{l}6.1 \text { vs. } 9.6 \\
(\mathbf{p}=<\mathbf{0 . 0 1})\end{array}$ & $\begin{array}{l}4.20 \text { vs. } \\
5.08 \\
(\mathbf{p}<\mathbf{0 . 0 0 1 )}\end{array}$ & & & & & & \\
\hline $\begin{array}{l}\text { Antero et al, } \\
2011\end{array}$ & 100 & & & $\begin{array}{l}3.35 \text { vs. } \\
2.81 \\
(p=0.1)\end{array}$ & & & & & & \\
\hline $\begin{array}{lll}\text { Haga } & \text { et } & \text { al, } \\
2012 & & \\
\end{array}$ & 100 & $\begin{array}{l}0.28 \text { vs. } \\
0.73 \\
(p=N S)\end{array}$ & $\begin{array}{l}1.1 \text { vs. } 2.2 \\
(p=N S)\end{array}$ & $\begin{array}{l}3.1 \text { vs. } 2.7 \\
(p=N S)\end{array}$ & & & & & & \\
\hline He J et al, 2013 & 100 & $\begin{array}{l}12.9 \text { vs. } \\
15.8 \\
(\mathbf{p}<\mathbf{0 . 0 5})\end{array}$ & $\begin{array}{l}12.1 \text { vs. } \\
14.5 \\
(\mathbf{p}=\mathbf{0 . 0 1 9 )}\end{array}$ & $\begin{array}{l}5.9 \text { vs. } 6.4 \\
(\mathbf{p}=\mathbf{0 . 0 0 9})\end{array}$ & & $\begin{array}{l}7.59 \text { vs. } 10.8 \\
(p=0.346)\end{array}$ & $\begin{array}{l}27.1 \text { vs. } 21.6 \\
(p=0.320)\end{array}$ & $\begin{array}{l}11.7 \text { vs. } 44.6 \\
(\mathbf{p}<\mathbf{0 . 0 0 1})\end{array}$ & $\begin{array}{l}4.81 \text { vs. } 14.9 \\
(\mathbf{p}=\mathbf{0 . 0 0 2})\end{array}$ & $\begin{array}{l}0.23 \text { vs. } 2.7 \\
(\mathbf{p}=\mathbf{0 . 0 1 0})\end{array}$ \\
\hline $\begin{array}{l}\text { Abdelghani et } \\
\text { al, } 2014\end{array}$ & 100 & $\begin{array}{l}3.7 \text { vs. } \\
3.2 \\
(p=0.4)\end{array}$ & $\begin{array}{l}6.2 \text { vs. } 5.6 \\
(p=0.4)\end{array}$ & $\begin{array}{l}4.13 \text { vs. } \\
4.05 \\
(p=0.8)\end{array}$ & $\begin{array}{l}1.5 \text { vs. } \\
27.2 \\
(\mathbf{p}=\mathbf{0 . 0 1})\end{array}$ & & & & & \\
\hline $\begin{array}{l}\text { Brown et al., } \\
2015\end{array}$ & 100 & & & $\begin{array}{l}4.3 \text { vs. } 3.2 \\
(\mathbf{p}=\mathbf{0 . 0 1})\end{array}$ & & & & & & \\
\hline \multicolumn{11}{|c|}{ SSc vs. SSc-sSS patients } \\
\hline Osial et al, 1983 & & & & & & & & $\begin{array}{l}\text { Unspecified } \\
\text { pulmonary } \\
\text { disease } \\
64 \text { vs. } 65\end{array}$ & 9 vs. 12 & \\
\hline $\begin{array}{l}\text { Avouac et al, } \\
2006\end{array}$ & & & & & & & & $\begin{array}{l}\text { Lung } \\
\text { fibrosis } \\
45 \text { vs. } 11 \\
\text { (p=0.02) } \\
\text { PAH } \\
19 \text { vs. } 11 \\
\text { (NS) }\end{array}$ & & \\
\hline $\begin{array}{l}\text { Salliot et al, } \\
2006\end{array}$ & & & & & $\begin{array}{l}94.7 \text { vs. } \\
92.6 \\
(p=0.98)\end{array}$ & & & $\begin{array}{l}\text { Lung } \\
\text { fibrosis } \\
29 \text { vs. } 11.1 \\
(\mathbf{p}=\mathbf{0 . 0 5}) \\
\text { PAH 15.1 } \\
\text { vs. } 7.4 \\
(p=0.60)\end{array}$ & $\begin{array}{l}14.9 \text { vs. } 3.7 \\
(\mathrm{p}=0.21)\end{array}$ & \\
\hline
\end{tabular}




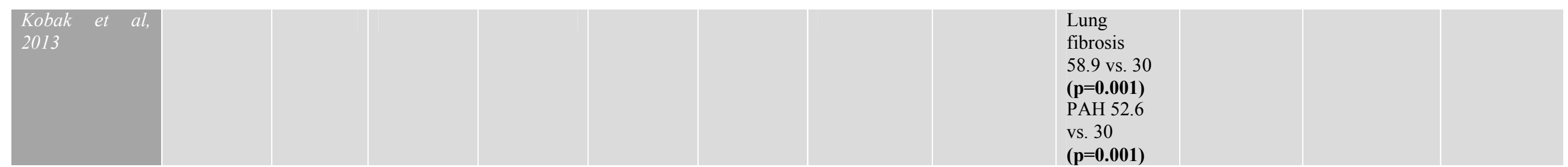

Legend: PAH - pulmonary arterial hypertension; NS - not significant; NPSLE- neuropsychiatric lupus.

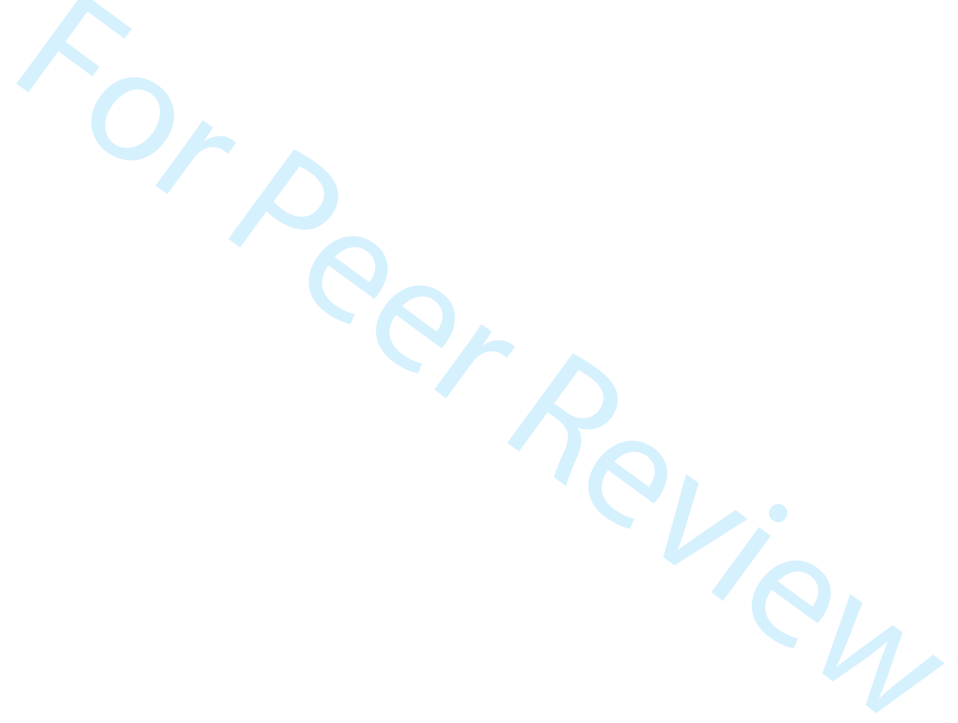




\begin{tabular}{|c|c|c|c|c|c|c|c|c|c|c|}
\hline Disease/Ref & $\begin{array}{l}\text { Ro/ SSA } \\
\text { antibodies } \\
\text { (\% } \\
\text { patients) }\end{array}$ & $\begin{array}{l}\text { La/ SSB } \\
\text { antibodies } \\
\text { (\% } \\
\text { patients) }\end{array}$ & $\begin{array}{l}\text { RF } \\
(\% \\
\text { patients/ } \\
\text { U/mL) }\end{array}$ & $\begin{array}{l}\text { ANA } \\
(\% \\
\text { patients })\end{array}$ & $\begin{array}{l}\text { dsDNA } \\
(\% \\
\text { patients })\end{array}$ & $\begin{array}{l}\text { Anti } \\
\text { CCP } \\
(\% \\
\text { patients })\end{array}$ & $\begin{array}{l}\text { Other antibodies/ } \\
\text { markers } \\
\text { (\% patients) }\end{array}$ & $\begin{array}{l}\text { ESR/CRP } \\
(\mathrm{mm} / \mathrm{h} ; \\
\mathrm{mg} / \mathrm{l})\end{array}$ & $\begin{array}{l}\text { Hyper } \\
\text { gamma } \\
\text { globulinaemia } \\
\text { (\% patients) }\end{array}$ & $\begin{array}{l}\text { Thrombo } \\
\text { Cytopaenia } \\
\text { (\% patients) }\end{array}$ \\
\hline \multicolumn{11}{|c|}{ SLE vs. SLE-sSS patients } \\
\hline $\begin{array}{l}\text { Nossent et } \\
\text { al, } 1998\end{array}$ & $\begin{array}{l}44 \text { vs. } 48 \\
(p=N S)\end{array}$ & $\begin{array}{l}38 \text { vs. } 33 \\
(p=N S)\end{array}$ & & $\begin{array}{l}88 \text { vs. } 87 \\
(p=N S)\end{array}$ & $\begin{array}{l}71 \text { vs. } 53 \\
(p=N S)\end{array}$ & & $\begin{array}{l}\text { Anticardiolipin } \\
33 \text { vs. } 41(\mathrm{p}=\mathrm{NS}) \\
\text { Anti-Sm } \\
21 \text { vs. } 15(\mathrm{p}=\mathrm{NS}) \\
\text { Anti-U1 nRNP } \\
26 \text { vs. } 25(\mathrm{p}=\mathrm{NS})\end{array}$ & & & $\begin{array}{l}9 \text { vs. } 26 \\
(\mathbf{p}<\mathbf{0 . 0 5})\end{array}$ \\
\hline $\begin{array}{l}\text { Uhlig et al, } \\
1999\end{array}$ & & & $\begin{array}{l}48.2 \mathrm{U} / \mathrm{mL} \\
\text { vs. } 62.2 \\
\mathrm{U} / \mathrm{mL} \\
(\mathrm{p}=0.08)\end{array}$ & & & & & $\begin{array}{l}\text { ESR } \\
18.6 \text { vs. } 26.8 \\
(\mathbf{p}<\mathbf{0 . 0 1}) \\
\text { CRP } \\
11.9 \text { vs. } 13.8 \\
(p=0.33)\end{array}$ & & \\
\hline $\begin{array}{l}\text { Gilboe et al, } \\
2001\end{array}$ & $\begin{array}{l}36 \text { vs. } 89 \\
(\mathbf{p}=\mathbf{0 . 0 5})\end{array}$ & $\begin{array}{l}11 \text { vs. } 56 \\
(\mathbf{p}=\mathbf{0 . 0 5})\end{array}$ & & & $\begin{array}{l}60 \text { vs. } 44 \\
(\mathbf{p}=\mathbf{0 . 0 5})\end{array}$ & & & & & \\
\hline $\begin{array}{l}\text { Manoussakis } \\
\text { et al, } 2004\end{array}$ & $\begin{array}{l}23.9 \text { vs. } \\
38.5 \\
(\mathbf{p}=\mathbf{0 . 0 0 8})\end{array}$ & $\begin{array}{l}7.0 \text { vs. } \\
38.5 \\
(\mathbf{p}=<\mathbf{0 . 0 0 1} \\
)\end{array}$ & $\begin{array}{l}28.6 \text { vs. } 64 \\
(\mathbf{p}<\mathbf{0 . 0 0 1})\end{array}$ & $\begin{array}{l}100 \% \text { both } \\
\text { groups }\end{array}$ & $\begin{array}{l}77.3 \text { vs. } \\
69.2 \\
(p=0.436)\end{array}$ & & $\begin{array}{l}\text { Anticardiolipin antibodies } \\
52.9 \text { vs. } 45.8(\mathrm{p}=0.639) \\
\text { Anti-U1 nRNP antibodies } \\
12.7 \text { vs. } 11.5(\mathrm{p}=0.999) \\
\text { Anti-Sm } \\
11.3 \text { vs. } 7.7(\mathrm{p}=0.999) \\
\text { Cryoglobulins } \\
14.7 \text { vs. } 15.8(\mathrm{p}=0.999)\end{array}$ & & & $\begin{array}{l}26.3 \text { vs. } 7.7 \\
(\mathbf{p}=\mathbf{0 . 0 3})\end{array}$ \\
\hline $\begin{array}{l}\text { Szanto et al, } \\
2006\end{array}$ & $\begin{array}{l}74 \text { vs. } \\
94.64 \\
(\mathbf{p}<\mathbf{0 . 0 1})\end{array}$ & $\begin{array}{l}44 \text { vs. } \\
73.21 \\
(\mathbf{p}<\mathbf{0 . 0 1})\end{array}$ & $\begin{array}{l}31.65 \mathrm{U} / \mathrm{m} \\
\mathrm{L} \text { vs. } \\
120.39 \\
\mathrm{u} / \mathrm{mL} \\
(\mathrm{p}=0.126)\end{array}$ & & $\begin{array}{l}223.35 \text { vs. } \\
132.51 \\
(\mathbf{p}<\mathbf{0 . 0 1})\end{array}$ & & $\begin{array}{l}\text { Anti-U1 nRNP } \\
41.6 \text { vs. } 37.1(p=0.603) \\
\text { Anti-Sm } \\
38.7 \text { vs. } 22.9(p=0.06)\end{array}$ & & & $\begin{array}{l}36 \text { vs. } 25 \\
(p=0.218)\end{array}$ \\
\hline
\end{tabular}




\begin{tabular}{|c|c|c|c|c|c|c|c|c|c|c|}
\hline $\begin{array}{l}\text { Pan et al, } \\
2008\end{array}$ & $\begin{array}{l}27.6 \text { vs. } \\
71.4 \\
(\mathbf{p}<\mathbf{0 . 0 0 1})\end{array}$ & $\begin{array}{l}17.4 \text { vs. } \\
51.4 \\
(\mathbf{p}<\mathbf{0 . 0 0 1})\end{array}$ & & $\begin{array}{l}74.4 \text { vs. } \\
85.7 \\
(p=0.13)\end{array}$ & $\begin{array}{l}38.3 \text { vs. } 60 \\
(\mathbf{p}=\mathbf{0 . 0 1 1})\end{array}$ & & & & & $\begin{array}{l}42 \text { vs. } 40 \\
(p=0.816)\end{array}$ \\
\hline $\begin{array}{l}\text { Baer et al, } \\
2010\end{array}$ & $\begin{array}{l}26.8 \text { vs. } \\
45.3 \\
(\mathbf{p}<\mathbf{0 . 0 0 1})\end{array}$ & $\begin{array}{l}10 \text { vs. } 22.1 \\
(\mathbf{p}<\mathbf{0 . 0 0 1})\end{array}$ & & & $\begin{array}{l}59.1 \text { vs. } \\
45.4 \\
(\mathbf{p}<\mathbf{0 . 0 0 1})\end{array}$ & & $\begin{array}{l}\text { Anticardiolipin } \\
49.1 \text { vs. } 41.7(\mathbf{p}=\mathbf{0 . 0 3}) \\
\text { Anti-U1 nRNP } \\
28 \text { vs. } 13.3(\mathbf{p}<\mathbf{0 . 0 0 1}) \\
\text { Anti-Sm } \\
17.3 \text { vs. } 9.7(\mathbf{p}=\mathbf{0 . 0 0 4})\end{array}$ & & & $\begin{array}{l}21.9 \text { vs. } 17.8 \\
(p=0.14)\end{array}$ \\
\hline \multicolumn{11}{|c|}{ RA vs. RA-sSS patients } \\
\hline $\begin{array}{l}\text { Antero et al, } \\
2011\end{array}$ & & & $\begin{array}{l}58 \text { vs. } 70 \\
(p=0.24)\end{array}$ & $\begin{array}{l}30.6 \text { vs. } 30 \\
(p=0.95)\end{array}$ & & $\begin{array}{l}70.3 \text { vs. } \\
75 \\
(p=1.0)\end{array}$ & & N/A & & \\
\hline $\begin{array}{l}\text { Haga et al, } \\
2012\end{array}$ & & & $\begin{array}{l}156.46 \text { vs. } \\
54.90 \\
(p=N S)\end{array}$ & & & $\begin{array}{l}136.89 \\
\mathrm{U} / \mathrm{mL} \text { vs. } \\
125.17 \\
\mathrm{U} / \mathrm{mL} \\
(\mathrm{p}=\mathrm{NS})\end{array}$ & & $\begin{array}{l}\text { ESR } \\
20.53 \text { vs. } \\
14.90(p=N S) \\
\text { CRP } \\
1.52 \text { vs. } 1.20 \\
(p=N S)\end{array}$ & & \\
\hline $\begin{array}{l}\text { He J et al, } \\
2013\end{array}$ & $\begin{array}{l}4.69 \text { vs. } \\
39.2 \\
(\mathbf{p}=\mathbf{0 . 0 0 1})\end{array}$ & $\begin{array}{l}1.39 \text { vs. } \\
14.9 \\
(\mathbf{p}=\mathbf{0 . 0 0 1 )}\end{array}$ & $\begin{array}{l}75.6 \mathrm{vs.} \\
95.7 \\
(\mathbf{p}=\mathbf{0 . 0 0 1})\end{array}$ & $\begin{array}{l}51.7 \text { vs. } \\
79.8 \\
(\mathbf{p}=\mathbf{0 . 0 0 1})\end{array}$ & & $\begin{array}{l}71.9 \text { vs. } \\
77.8 \\
(p=0.5)\end{array}$ & & $\mathrm{N} / \mathrm{A}$ & $\begin{array}{l}\operatorname{IgA} \\
33.6 \text { vs. } 48.5 \\
(\mathbf{p}=\mathbf{0 . 1 0 6}) \\
\operatorname{IgG} \\
35.6 \text { vs. } 54.3 \\
(\mathbf{p}=\mathbf{0 . 0 1 0}) \\
\text { IgM } \\
10.6 \text { vs. } 11.8 \\
(p=0.951)\end{array}$ & $\begin{array}{l}0.5 \text { vs. } 9.5 \\
(\mathbf{p}=\mathbf{0 . 0 0 1})\end{array}$ \\
\hline $\begin{array}{l}\text { Abdelghani } \\
\text { et al, } 2014\end{array}$ & $\begin{array}{l}1.5 \text { vs. } 0 \\
(p=1)\end{array}$ & & $\begin{array}{l}81.8 \text { vs. } \\
67.1 \\
(p=0.5)\end{array}$ & $\begin{array}{l}53.8 \text { vs. } \\
63.6(p=0.1)\end{array}$ & & $\begin{array}{l}64 \text { vs. } 90 \\
(p=0.1)\end{array}$ & $\begin{array}{l}\text { B2-m serum mean level } \\
1.9 \mathrm{mg} / \mathrm{l} \mathrm{vs} .2 .4 \\
\mathrm{mg} / \mathrm{l}(\mathrm{p}=0.02)\end{array}$ & $\begin{array}{l}\text { ESR } \\
20.9 \text { vs. } 24.8 \\
(p=0.4) \\
\text { CRP } \\
14 \text { vs. } 12.6\end{array}$ & & \\
\hline
\end{tabular}




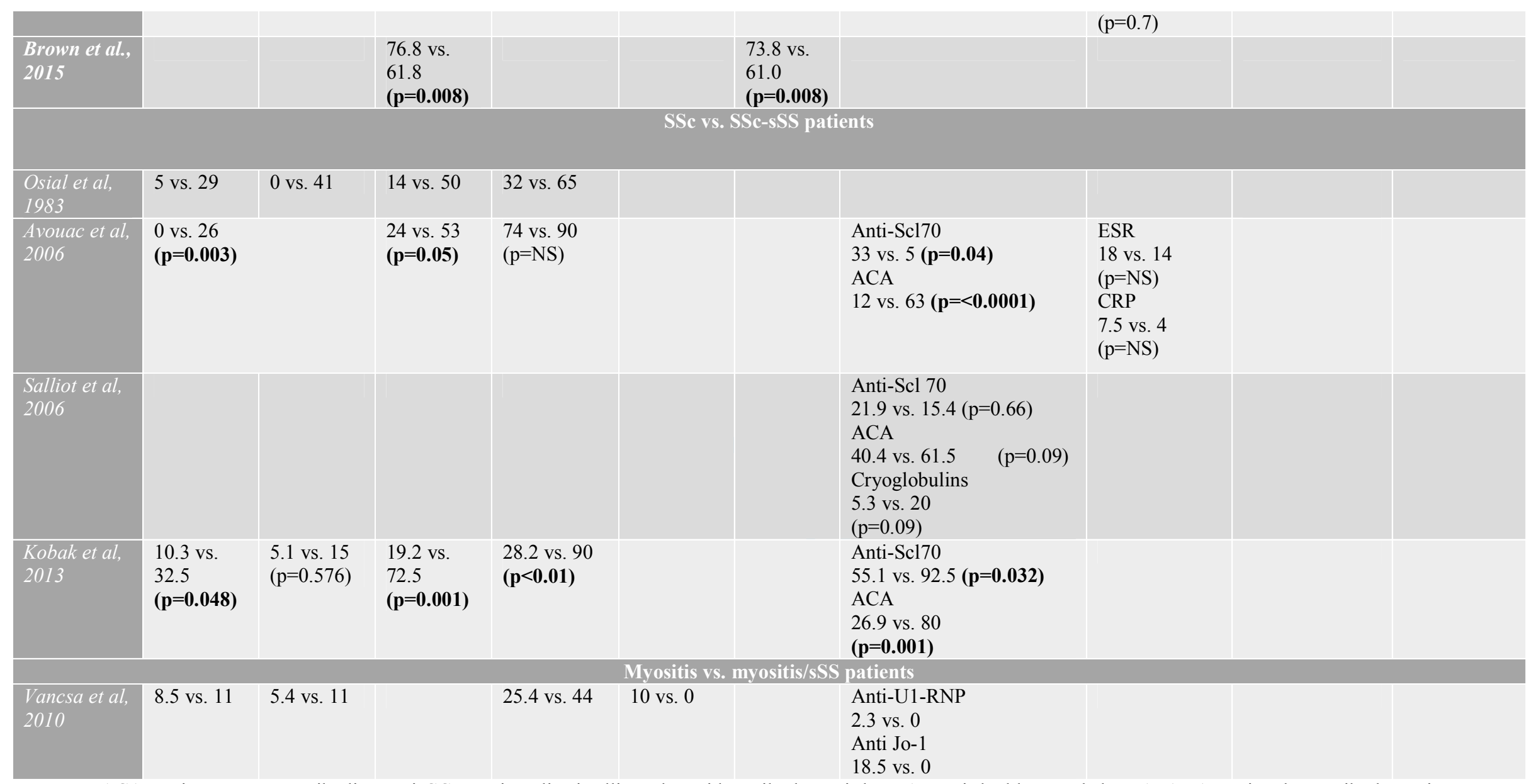

Legend: ACA- anti-centromere antibodies; anti-CCP- anti-cyclic citrullinated peptide antibody; anti-dsDNA - anti-double stranded DNA; ANA- antinuclear antibody; antiSc170 - anti-topoisomerase antibody; anti-Sm - anti-smith antibody; anti-U1-RNP - anti-nuclear ribonucleoprotein antibody; B2-m - beta 2 microglobulin; RF-Rheumatoid factor. 


\section{Systematic review and meta-analysis of the epidemiology of}

polyautoimmunity in Sjögren's syndrome (secondary Sjögren's syndrome) focusing on autoimmune rheumatic diseases

\section{Submission type: article}

Short title: Polyautoimmunity in Sjögren's syndrome

\section{Authors:}

Hudaifa Alani', Julian R. Henty ${ }^{2}$, Nicolyn L Thompson ${ }^{3}$, Elizabeth Jury ${ }^{3}$ and Coziana Ciurtin ${ }^{3 *}$

${ }^{1}$ Kettering General Hospital, NN16 8UZ, Kettering, United Kingdom

${ }^{2}$ Department of Medical Physics, University College London, London, United Kingdom

${ }^{3}$ Department of Rheumatology, University College London, London, United Kingdom 


\section{*Corresponding author:}

Dr. Coziana Ciurtin, PhD, FRCP, Department of Rheumatology, University College London, 250 Euston Road, London, NW1 2PG, email: c.ciurtin@ucl.ac.uk, phone: +44(0)2034479035, fax: +44(0)20344797268 . 


\section{Abstract}

Objective: The epidemiology of pelyimmunitypolyautoimmunity in Sjögren's syndrome (secondary Sjögren's syndrome - sSS) is not welldefined and was not investigated before using a systematic approach. We conducted a systematic review of the epidemiology of sSS associated with rheumatoid arthritis (RA), systemic lupus erythematosus (SLE), scleroderma and myositis, assessing the prevalence rates (PRs) and clinical and serological features of sSS.

Methods: A systematic literature search of PubMed and Embase databases (updated to March 2016) was performed to identify all published data on prevalence rate, demographic profile, clinical manifestations, laboratory features and causes of death associated with sSS. The prevalence rates of pSS were summarised with PRs and 95\% CIs.

Results: The literature search identified 1639 citations, out of which 42 fulfilled the inclusion criteria. Only 19 studies had moderate to good quality and were selected for the meta-analysis. According to a random-effects model, the pooled PR for sSS associated with RA was 19.5\% ( $95 \%$ CI 11.2 to 27.8 ) and the pooled PR for sSS associated with SLE was $13.96 \%$ (95\% CI 8.88 to 19.04). The female/male ratio of sSS in the RA population was 14.7 (95\% CI 7.09 to 256) and 16.82 (95\% CI 1.22 to 32.4) in the SLE population.

Conclusion: Prevalence rates of sSS vary widely in different populations. Both meta-analyses conducted in the RA and SLE populations were characterised by a high degree of study heterogeneity. The results of this meta-analysis highlighted the need for better quality population studies.

Keywords: secondary Sjögren's syndrome, polyimmunitypolyautoimmunity in Sjögren's syndrome, prevalence, sex ratio, systematic review, meta-analysis. 


\section{Introduction}

Sjögren's syndrome (SS) is a chronic systemic autoimmune disorder, which is associated in the majority of cases with lymphocytic infiltration of exocrine glands and epithelium, feature that is considered the histological hallmark of the disease (1). The T cell mediated attack on salivary and lacrimal glands results in chronic inflammation, which is considered in the majority of cases the leading cause tote glandular atrophy and deficient glandular function (2). Although, the temporal relationship between the presence of glandular inflammatory infiltrate and atrophic/fibrotic changes associated with ageing and/or disease progression is difficult to appreciate, the minor salivary gland biopsy have their role in the stratification and prognostication of patients with SS (3).

SS is characterised clinically by symptoms of dry mouth (xerostomia) and dry eyes (xerophthalmia), known as sicca symptoms. SS can progress to affect many organ systems (lung, kidney, gastro-intestinal tract, skin, musculoskeletal, and peripheral and rarely central nervous system), and as a result, other clinical manifestations ranging from mild to more severe disease may occur, including: arthralgias, vasculitis, peripheral neuropathy, renal failure and interstitial lung disease (4). In addition, SS is associated with increased risk of lymphoma (5).

SS can occur either alone as primary SS (pSS) or in association with another well-defined autoimmune condition, such as systemic lupus erythematosus (SLE), rheumatoid arthritis (RA), systemic sclerosis (SSc), or dermatomyositis (DM), in which case it is known as secondary SS (sSS) (6). Although, some researchers prefer to use the term of "polyauto-immunity" associated with SS rather than sSS $(7,8)$, for the purpose of our systematic review, we used the previously accepted terminology of sSS, which enabled us to identify all the relevant papers. In addition, the term "polyautoimmunity" refers to clusters of autoimmune conditions, which may include or not autoimmune rheumatic diseases (8).-Previous papers focused predominantly on prevalence studies and distinct immunologic differences between different sSS subtypes, rather than 
differences in clinical presentations or controversies regarding the patients' diagnosis.

The diagnosis of SS cannot be made on a single test or symptom. The variability in presentation of SS has led to a difficulty over the years in establishing universally accepted classification criteria. Therefore, there has been a variation in prevalence estimates in the few epidemiological studies that have been documented, as they used different classification criteria $(1,9)$. Although the clinical features of pSS are relatively wellresearched in large epidemiological studies $(10,11)$, there are very few studies looking at the epidemiology and clinical and serological features of sSS. The authors felt that a systematic review of the epidemiology of sSS was needed in order to establish any significant differences in the presentation of sSS according to the background rheumatic condition of patients that might have impact on their long-term management.

Over the last few decades, diagnostic criteria have varied according to different national and international groups. At present, the most widely accepted and cited criteria are the American-European consensus group classification criteria (AECG), which were published in 2002 as a revision from the original European Study group criteria described in $1996(2,12)$. Interestingly, the presence of anti-Ro/La antibodies has not been included as a mandatory classification criteria for sSS, since it was not shown to be significant in previous analysis (13). New classification criteria for SS were recently developed and validated $(14,15)$. Although, they do not introduce any significant changes, they have been validated in three international patient cohorts, and emphasised the role of expert opinion in diagnosing SS.

\section{Methodology}

We performed a PUBMED and EMBASE search for articles involving humans only, published between 1984 and 2016. The MESH terms used were: secondary Sjögren's, epidemiology of Sjögren's syndrome, secondary Sjögren's and systemic lupus erythematosus, secondary Sjögren's and rheumatoid arthritis, secondary Sjögren's and systemic sclerosis/scleroderma/CREST syndrome, and secondary Sjögren’s and myositis. As 
criteria for study selection, we considered all the studies on the epidemiology, diagnosis and follow-up of patients with sSS. We excluded editorials, commentaries, animal studies, questionnaire studies, case reports, case-series, and studies of treatment (Figure 1).

We extracted data on prevalence, demographic profile, clinical manifestations, laboratory features, underlying autoimmune diseases and causes of death from the selected articles, where available, and organised them in tables.

Studies were grouped according to the following patient categories: 1). secondary Sjögren's and systemic lupus erythematosus, 2). secondary Sjögren's and rheumatoid arthritis, 3). secondary Sjögren's and scleroderma and 4). secondary Sjögren's and myositis. We present our results data under the following headings: prevalence and demographics of secondary Sjögren's syndrome in different autoimmune diseases, clinical and laboratory features of Sjögren's syndrome in different autoimmune diseases, and morbidity and mortality associated with secondary Sjögren's syndrome. Prevalence rates were calculated using 95\% CI. Pooled prevalence rates and sex ratios were calculated using a random effects model (based on the Q and $\mathrm{I}^{2}$ tests of heterogeneity among studies).

\section{Results:}

The initial research yielded 1639 articles, which were screened for titles and abstracts, of which 37 were selected for review based on the inclusion and exclusion criteria mentioned above (the detailed process of paper selection is detailed in Figure 1). Following an additional manual search of other relevant articles, we identified 42 full papers and abstracts, which met the inclusion criteria and were analysed further.

We appreciate a risk of reporting bias for the majority of studies as they addressed different populations and used different classification criteria for sSS. The study quality was weighted as poor, moderate and good based on the following criteria: number of patients, the use of established SS classification criteria, data about patient sex, ethnicity and disease duration, inclusion of lip biopsy in the classification criteria (especially as the serology is likely to be positive in lupus patients, irrespective of concomitant SS). The assessment of study quality was reviewed independently by HA and CC. There was an $82 \%$ consensus. The studies in which case the consensus was not reached, were further evaluated by 
the third author (EJ) and graded based on the assessment made by two of the three authors. The details of study quality assessment were included in Table 1. Based on this selection, 9 SLE studies and 11 RA studies assessed as having moderate to good quality were included in the final meta-analysis.

\section{Prevalence and demographics of SS associated with different autoimmune diseases}

Our search identified 40 worldwide studies, which evaluated the prevalence of sSS in patients with RA, SLE, SSc and myositis. The study designs included were retrospective, cross-sectional and prospective (Table 2). The number of patients used in each study ranged from 6 to 2694.

\section{Criteria}

Different criteria were used across the different studies to classify patients as having sSS, including AECG (14), ECC (7), Japanese classification criteria (1), and other criteria (9). Nine studies did not specify the classification criteria they used.

\section{Prevalence}

We found 18 studies which looked at the prevalence of RA-sSS (between 1987-2013) (16-33), 13 for SLE-sSS (1998-2015) (34-46), 6 for SScSSS (1983-2013) (47-52) and 3 for myositis-sSS (2011-2014) (53-55). The prevalence ranged from 3.6\%-55\% for RA-sSS, 5\%-22\% SLE-sSS, $14 \%-60 \%$ SSc-sSS and 10-23\% for myositis-sSS. In the RA-sSS studies three of the highest reported prevalence all came from studies carried out in Greece on Greek patients $(31-39.8 \%)(18,20,23)$.

\section{Gender}

Not all studies reported the gender of the patients, however in the 15 that did, females were predominantly affected (82\%-100\%). Less than half of all studies (17/37) highlighted the ethnicity of the patients included in the study. 
Age

In five studies $(36,39,42,44,45)$ SLE-sSS patients were reported to be significantly older when compared to those with SLE only (48.3 vs. $36.1, \mathrm{p}<0.001 ; 41.3$ vs. $35.8, \mathrm{p}=0.003 ; 50.8$ vs. $43.6, \mathrm{p}=0.01 ; 41$ vs. $35 \mathrm{p}=0.03$, and 49.5 vs. $41.4 \mathrm{p}<0.001$ ). This was not found to be the case for RA-sSS patients when compared to RA patients as reported by two studies (63.0 vs. 59.2, p=0.33 and 66.36 vs. 62.40, p=NS) (21, 28). There was also no significant difference in age between SSc vs. SSc-sSS patients reported in three studies (56 vs. 54, p=NS; 50.2 vs. 55, p=0.18, and 48.3 vs. $50.5 \mathrm{p}=\mathrm{NS})(50-52)$.

\section{Disease duration}

Disease duration was reported in 26/40 studies and varied across all studies ranging from 4 months -12 years for RA-sSS (16, 20-23, 25, 27-29, 32, 33), 3-46 years SLE-sSS (34-37, 39, 41, 42, 44), 7-8 years SSc-sSS (47, 50-52), and 67 months to 20 years for myositis-sSS (53-55).

Only the RA-sSS studies looked into the relationship between disease duration and sSS incidence rate. There were conflicting reports with regard to whether RA disease duration plays any role in the reported occurrence rate of sSS. Two studies found that the cumulative prevalence of sSS did increase with RA duration $(19,29)$. This finding however, was not supported by four other studies $(21,22,25,28)$.

\section{Clinical and laboratory features of sSS associated with different autoimmune diseases:}

We identified 17 studies assessing the clinical and laboratory features of sSS (Tables 3-4). The reported clinical features varied depending on which condition associated with sSS was being looked at. 


\section{SLE-SSS}

Of the seven studies $(36,37,39,42,44-46)$ assessing clinical features of SLE-sSS, the majority reported data on the presence of renal involvement and central nervous system (CNS) involvement. Renal involvement was found to be significantly reduced in SLE-sSS patients across five studies $(36,37,39,44,45)$, while thyroiditis was found to be significantly higher. CNS involvement prevalence, although reduced in SLE-sSS patients, was not significant in any of the three studies. Only one study reported no cases of lymphoma in their cohort of 26 SLE-sSS patients (36), although they reported 8 cases of lymphoma in their pSS comparative group $(\mathrm{N}=86)$.

Regarding serological markers, only anti-Ro/SSA and anti-La/SSB antibodies were significantly raised in SLE-sSS compared to SLE patients in five studies $(36,37,39,42,44)$. Anti-dsDNA was higher in SLE patients and this reached significance in four studies (37, 39, 42, 44). Thrombocytopenia was lower in SLE-sSS patients, although this was only significant in two studies $(36,45)$.

Koskenmies et al. reported that sSS was most commonly observed in patients with subacute cutaneous lupus erythematosus (SCLE) and SLE than in patients with discoid lupus erythematosus ( $16.4 \%$ vs. $22.1 \%$ vs. $2.3 \%, p<0.001)(43)$.

\section{RA-SSS}

Five studies addressed clinical features of RA-sSS patients $(21,22,25,27,28)$. The majority reported data on joint swelling, tender joints and disease activity score assessing 28 joints (DAS 28 score). Half the studies reported that tender joints were significantly higher in RA-sSS patients compared to RA patients $(22,27)$, while the other half reported no significant difference $(21,28)$. Only one reported study looked at other clinical features including lymphadenopathy, thyroiditis, lung, renal and CNS involvement (27). 
Laboratory analysis was mainly focused on RF, anti-CCP, CRP and ESR levels. There was inconsistency concerning the reported RF levels. Three studies $(22,25,27)$ reported higher levels of RF in RA-sSS patients, one being significant, while the opposite was found in two other studies, although not significant $(21,28)$.

\section{$S S c-s S S$}

Four studies $(47,50-52)$ reported data about lung involvement, mainly pulmonary fibrosis (PF) and pulmonary arterial hypertension (PAH). PF occurred significantly less frequently in SSc-sSS patients. Only one study reported lower occurrence of PAH in SSc-sSS, which was significant (52).

The majority of studies looked at anti-topoisomerase 1 antibodies and anti-centromere (ACA) levels. ACA levels were significantly higher in SSc-sSS patients across all three studies (50-52). However, there was discrepancy regarding the prevalence of anti-topoisomerase 1 antibodies.

\section{Myositis-SSS}

In one study, all six patients with both diseases presented with a pattern of muscle weakness typical of IBM (53). Five IBM-sSS patients were treated with prednisolone and methotrexate, four of whom had temporary symptomatic improvement (6-24 months). This was a far greater response in comparison to the IBM only group in whom only $27 \%$ had a transient response to treatment.

Immunogenetics

\section{SLE-SSS}


Immunogenetic analysis was carried out in two studies. In one study there was no significant difference between the SLE and SLE-sSS patients when looking at the HLA alleles (42). However, in another study, the HLA associations distinguished the SLE group from those with SLE-sSS. Those with SLE were found to have increased phenotype and allele frequencies for DRB $1 * 1501$ ( $\mathrm{p}=0.020$ and $\mathrm{p}=0.015$, respectively) and DQB1*0602 (both $\mathrm{p}<0.001$ ) that was significant (36).

\section{Myositis-sSS}

In the study mentioned above (53), all six patients carried the HLA-DRB1*0301 allele or its equivalent HLA-DR3 serological specificity. They also carried either all or some of the major markers of the $8.1 \mathrm{MHC}$ ancestral haplotype. This allele was also reported to be highly prevalent among the Norwegian patients included in another study (25\%), which found a prevalence of rheumatic disorders of $24 \%$, which is twice as high as previously reported (55).

\section{Morbidity and mortality associated with sSS}

In a few RA studies other aspects of the disease including its effect on quality of life and its involvement in haematological malignancies was studied.

Health status

\section{RA-sSS}

In three of the five studies measuring health status there was no difference in the DAS-28 mean scores in RA-sSS compared to RA patients (21, $25,28)$. In two other studies $(22,27)$ however, a higher DAS-28 score was found in RA-sSS patients, compared to RA patients $(6.44$ vs. 5.96 , $\mathrm{p}=0.02$ and 5.08 vs. $4.20, \mathrm{p}=<0.001$, respectively). 
One study (22) looked at other health status measures including pain visual analogue scale (VAS) scale, fatigue VAS and Modified Health Assessment Questionnaire (M-HAQ), and found that the RA-sSS patients scored significantly higher in all three tests in comparison to RA patients ( 43.1 vs. $32.9, \mathrm{p}=<0.01 ; 49.8$ vs. $39.7, \mathrm{p}=0.03$, and 1.75 vs. $1.55, \mathrm{p}=0.04$, respectively). Another study (28) looked at both M-HAQ and pain VAS tests and reported similar findings in both groups of patients ( 0.84 vs. 0.81 , $\mathrm{p}=0.7$, and 35.9 vs. 42.4 , $\mathrm{p}=0.3$, respectively).

\section{Haematological malignancy and mortality}

\section{SLE-SSS}

Nossent et al. (45) reported a significantly reduced overall mortality in patients with SLE-sSS compared to SLE patients (4\% vs. $13.5 \%$, $\mathrm{p}=0.01$ ). In two studies in which patients where followed up for three years and 8 years respectively, none of the patients developed lymphoma $(36,45)$.

Martens et al. looked at the survival of sSS patients in a population-based sample in Minnesota, USA between 1976 and 1992 . Of the 74 cases $24(33 \%)$ had sSS and 50 (67) had pSS. It found that when compared with the general population, SS patients had increased mortality ( $\mathrm{p}=0.04)$. Furthermore, when studied separately, the mortality was increased in sSS compared to pSS patients $(p=<0.005)$, with the majority of $s S S$ patients having associated RA ( $\mathrm{p}=0.86)(56)$.

\section{RA-SSS}


With regard to haematological malignancies, studies have reported increased incidence of non-Hodgkin's lymphoma (NHL) in RA-sSS patients. A Finnish study carried out by Kauppi et al. compared the incidence of NHL in 9,469 RA patients and 709 sSS patients. This study found the incidence of NHL to be almost two-fold in patients with RA-sSS (8.7, CI=4.3-1.6) compared to RA patients (4.5, CI=1.5-11) (56).

\section{$S S C-S S S$}

Baldini et al. described a new clinical phenotype of "ACA-positive limited scleroderma/SS overlap syndrome", which in their retrospective study was characterised by a benign SSc clinical course but at a high risk of non-Hodgkin's lymphoma (57).

\section{Meta- analysis of prevalence rates and sex ratios of SSS associated with SLE and RA}

The results of the meta-analysis revealed a pooled prevalence of $13.96 \%$ (95\% CI 8.88 to 19.04 ) in the SLE population and $19.5 \%$ (95\% CI 11.2 to 27.8) in the RA population. The statistical analysis of the selected studies revealed a high degree of heterogeneity as expected $\left(\mathrm{I}_{2}=99.98\right.$ for the SLE studies and 99.92 for the RA studies; therefore, we used a random effect statistical model for calculating the pooled prevalence). The results of the meta-analysis are presented in Figures 2 and 3.

We also analysed the sex ratio of sSS patients in the SLE and RA populations, which revealed a clear predominance of female patients (four RA studies reported that all their sSS/RA patients were females). The female: male ratios were 16.82 (95\% CI 1.22 to 32.4) for the SLE-sSS patients and 14.7 (95\% CI 7.09 to 256) for the RA-sSS population.

\section{Discussions}


Secondary SS is characterised by a heterogeneity of clinical manifestations, serological markers and symptoms, which are influenced by patients' underlying pathology. Unfortunately, large prospective studies comparing patients with sSS associated with different autoimmune rheumatic diseases are lacking. Previous studies were interested in comparing the clinical and laboratory features of an autoimmune disease alone or associated with sSS (as the majority of the studies included in this systematic review), or aimed to compare the epidemiology of pSS vs. sSS (this was beyond the scope of our systematic analysis). Other papers explored the communality of serological abnormalities and shared clinical picture in distinct autoimmune rheumatic diseases, such as RA and pSS (58), or advocated that the association of SS with multiple autoimmune diseases, is better described as "polyimmunitypolyautoimmunity" (7), as discussed in introduction. However, this terminology is not particularly exact in relation to the presence of clinical and serological features of SS in the context of rheumatic conditions (which is the focus of this review), as it also refers to associations of SS with other autoimmune diseases (8)-.

There is evidence of a great degree of heterogeneity within all these populations; although previous research established that pSS is associated with a different disease phenotype compared to sSS: e.g. pSS patients had a higher frequency of parotid gland enlargement and Ro and La antibody positivity (59), or had significantly higher levels of IL-2 and IL-6 in their saliva (60). An old study also suggested that extraglandular features are more common in pSS compared to sSS (61).

Our study could not address any controversies regarding the accuracy of patients' diagnosis (e.g. many clinicians' might decide based on their expert opinion to diagnose a patient as having RA associated with SS rather than symmetrical polyarthritis in the context of primary SS). In addition, our systematic review does not imply that patients with sSS associated with different ARDs have similar features (e.g. SLE and SS patients might have similar clinical presentation, which is not the case with patients with SS associated with SD or myositis). Even if only moderate-good quality studies were included in the meta-analysis, the studies with poor quality were also detailed in the paper. In addition, our 
systematic review also reported on papers relevant for the article theme, even if not selected by our systematic approach, such as papers referring to patients defined as having overlapping syndromes rather than sSS (57) or which contained no data about sSS prevalence (62).

Our analysis revealed that SSS is more common in women, irrespective of the underlying autoimmune disease. The confidence intervals of sex ratios in the SLE and RA patients with associated sSS reflected again the high heterogeneity of the studies and the inclusion of studies, which reported the presence of sSS exclusively in the female population.

Despite the effort to define the sSS patient population better, clinicians are still unable to answer practical questions regarding the difference in the long term outcome of sSS associated with an autoimmune disease compared to having only an autoimmune disease, or regarding the best way to stratify these patients to enable the choice of the most suitable therapeutic options. It is recognized that sSS is characterised by significant amount of variability in clinical presentation, which is influenced by the concomitant autoimmune disease; however, different studies reported various epidemiological features in the context of similar background autoimmune disease. This variation can be in part explained by the SS classification criteria applied, as well as patient selection criteria, their ethnicity or genetic background, and possible reporting bias (45, 63). Our results show that Raynaud's phenomenon, thyroiditis and Ro, La antibody positivity seemed to be more frequent in SLE-sSS patients compared to SLE group, while renal involvement and presence of dsDNA, and anti Sm antibodies were more common in the latter group. A less clearly defined trend was identified in the case of thrombocytopaenia and lung involvement, while the CNS involvement was reported in only one study as more frequent in the SLE-sSS group.

The number of tender and swollen joints was more commonly reported in the RA compared to RA-sSS group, however, the RA-sSS group had more CNS involvement, Raynaud's phenomenon, thrombocytopaenia and hypergammaglobulinaemia. 
In the scleroderma and myositis groups, the main differences were seen in the positivity of antibodies (the disease characteristic ones being more prevalent in their corresponding group). However, as there was no significant overlap between the clinical features of these autoimmune conditions, and the quality of the studies was poor, no assumptions can be made about the difference in the clinical presentation of SSc-sSS made for patients' clinical phenotype, apart from additional symptoms of dryness, and a possible subset of patients with overlap syndrome with milder disease presentation (57).

Importantly, studies of both SLE and SSc populations hinted at the possibility that these patients have the highest risk of lymphoma of all the autoimmune disease groups $(44,57)$.

Our systematic review included mainly prevalence studies (as the large population studies were lacking) and reported prevalence rather than incidence figures (as SSS is reported in patient groups rather than general population, and the appreciation of the newly diagnosed cases per year necessitates long prospective studies not available in the literature). The quality of the studies included in the analysis was poor to moderate because of the following reasons: significant heterogeneity of patient inclusion criteria (12 studies did not use validated classification criteria and were excluded from the final analysis); variable number of patients included (from 6 to 2694); different proportion of patients classified as having SS based on salivary gland biopsy (14 studies included the biopsy as a classification criteria for all the sSS patients, and 5 studies for a variable proportion of patients); limited geographic areas (only one study evaluated patients from two different countries), and difficulty to extrapolate data to other populations (there were no studies from South America, Africa or Oceania). There was also evidence of significant statistical heterogeneity in our meta-analysis of sSS prevalence in SLE and RA patients, probably due to both, clinical and methodological differences between studies.

The authors identified an unmet need for a consensus regarding the diagnostic/classification criteria for sSS in the context of different underlying autoimmune diseases, especially in the group of SLE/sSS, which is characterised by shared clinical and serological features that make the diagnosis difficult in the absence of a positive salivary gland biopsy. 
In conclusion, this is the first systematic review and meta-analysis of the epidemiology of sSS, which aimed to evaluate the characteristics of this heterogeneous population. Because of the lack of prospective longitudinal data in large population studies, there are still unanswered questions related to the malignancy risk of these patients or their clinical and laboratory features in less common autoimmune diseases.

Acknowledgement: CC was funded by a British Research Council grant (BRC-III/003).

\section{References:}

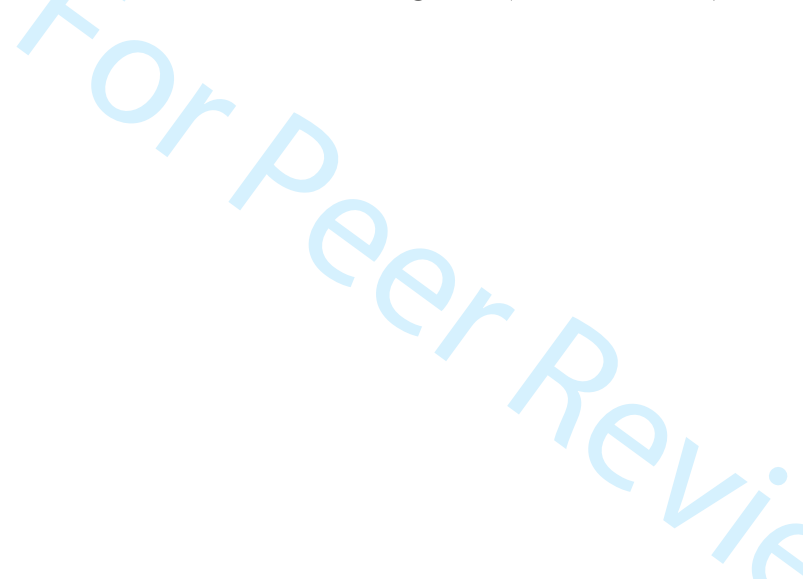

1. Baldini C, Talarico R, Tzioufas AG, Bombardieri S. Classification criteria for Sjogren's syndrome: a critical review. Journal of autoimmunity. 2012;39(1-2):9-14.

2. Jonsson R, Vogelsang P, Volchenkov R, Espinosa A, Wahren-Herlenius M, Appel S. The complexity of Sjogren's syndrome: novel aspects on pathogenesis. Immunol Lett. 2011;141(1):1-9.

3. Fisher BA, Brown RM, Bowman SJ, Barone F. A review of salivary gland histopathology in primary Sjogren's syndrome with a focus on its potential as a clinical trials biomarker. Ann Rheum Dis. 2015;74(9):1645-50.

4. Patel R, Shahane A. The epidemiology of Sjogren's syndrome. Clinical epidemiology. 2014;6:247-55. 
5. Johnsen SJ, Brun JG, Goransson LG, Smastuen MC, Johannesen TB, Haldorsen K, et al. Risk of non-Hodgkin's lymphoma in primary Sjogren's syndrome: a population-based study. Arthritis care \& research. 2013;65(5):816-21.

6. Theander E, Jacobsson LT. Relationship of Sjogren's syndrome to other connective tissue and autoimmune disorders. Rheum Dis Clin North Am. 2008;34(4):935-47, viii-ix.

7. Anaya JM, Rojas-Villarraga A, Mantilla RD, Arcos-Burgos M, Sarmiento-Monroy JC. Polyautoimmunity in Sjogren Syndrome. Rheum Dis Clin North Am. 2016;42(3):457-72.

8. Amador-Patarroyo MJ, Arbelaez JG, Mantilla RD, Rodriguez-Rodriguez A, Cardenas-Roldan J, Pineda-Tamayo R, et al. Sjogren's syndrome at the crossroad of polyautoimmunity. J Autoimmun. 2012;39(3):199-205.

9. Alamanos Y, Tsifetaki N, Voulgari PV, Venetsanopoulou Al, Siozos C, Drosos AA. Epidemiology of primary Sjogren's syndrome in north-west Greece, 1982-2003. Rheumatology. 2006;45(2):187-91.

10. Maldini C, Seror R, Fain O, Dhote R, Amoura Z, De Bandt M, et al. Epidemiology of primary Sjogren's syndrome in a French multiracial/multiethnic area. Arthritis care \& research. 2014;66(3):454-63.

11. Qin B, Wang J, Yang Z, Yang M, Ma N, Huang F, et al. Epidemiology of primary Sjogren's syndrome: a systematic review and meta-analysis. Annals of the rheumatic diseases. 2015;74(11):1983-9.

12. Vitali C, Bombardieri S, Jonsson R, Moutsopoulos HM, Alexander EL, Carsons SE, et al. Classification criteria for Sjogren's syndrome: a revised version of the European criteria proposed by the American-European Consensus Group. Ann Rheum Dis. 2002;61(6):554-8.

13. Vitali C, Bombardieri S, Moutsopoulos HM, Balestrieri G, Bencivelli W, Bernstein RM, et al. Preliminary criteria for the classification of Sjogren's syndrome. Results of a prospective concerted action supported by the European Community. Arthritis Rheum. 1993;36(3):340-7.

14. Shiboski CH, Shiboski SC, Seror R, Criswell LA, Labetoulle M, Lietman TM, et al. 2016 American College of Rheumatology/European League Against Rheumatism classification criteria for primary Sjogren's syndrome: A consensus and data-driven methodology involving three international patient cohorts. Ann Rheum Dis. 2017;76(1):9-16.

15. Shiboski CH, Shiboski SC, Seror R, Criswell LA, Labetoulle M, Lietman TM, et al. 2016 American College of Rheumatology/European League Against Rheumatism Classification Criteria for Primary Sjogren's Syndrome: A Consensus and Data-Driven Methodology Involving Three International Patient Cohorts. Arthritis Rheumatol. 2017;69(1):35-45.

16. Turesson C, O'Fallon WM, Crowson CS, Gabriel SE, Matteson EL. Extra-articular disease manifestations in rheumatoid arthritis: incidence trends and risk factors over 46 years. Ann Rheum Dis. 2003;62(8):722-7.

17. Calguneri M, Ureten K, Akif Ozturk M, Onat AM, Ertenli I, Kiraz S, et al. Extra-articular manifestations of rheumatoid arthritis: results of a university hospital of 526 patients in Turkey. Clin Exp Rheumatol. 2006;24(3):305-8.

18. Drosos AA, Lanchbury JS, Panayi GS, Moutsopoulos HM. Rheumatoid arthritis in Greek and British patients. A comparative clinical, radiologic, and serologic study. Arthritis Rheum. 1992;35(7):745-8.

19. Carmona L, Gonzalez-Alvaro I, Balsa A, Angel Belmonte M, Tena X, Sanmarti R. Rheumatoid arthritis in Spain: occurrence of extra-articular manifestations and estimates of disease severity. Ann Rheum Dis. 2003;62(9):897-900. 
20. Ioannidis JP, Tarassi K, Papadopoulos IA, Voulgari PV, Boki KA, Papasteriades CA, et al. Shared epitopes and rheumatoid arthritis: disease associations in Greece and meta-analysis of Mediterranean European populations. Semin Arthritis Rheum. 2002;31(6):361-70.

21. Haga HJ, Naderi Y, Moreno AM, Peen E. A study of the prevalence of sicca symptoms and secondary Sjogren's syndrome in patients with rheumatoid arthritis, and its association to disease activity and treatment profile. Int J Rheum Dis. 2012;15(3):284-8.

22. Uhlig T, Kvien TK, Jensen JL, Axell T. Sicca symptoms, saliva and tear production, and disease variables in 636 patients with rheumatoid arthritis. Ann Rheum Dis. 1999;58(7):415-22.

23. Andonopoulos AP, Drosos AA, Skopouli FN, Acritidis NC, Moutsopoulos HM. Secondary Sjogren's syndrome in rheumatoid arthritis. J Rheumatol. 1987;14(6):1098-103.

24. Mattey DL, Gonzalez-Gay MA, Hajeer AH, Dababneh A, Thomson W, Garcia-Porrua C, et al. Association between HLA-DRB1*15 and secondary Sjogren's syndrome in patients with rheumatoid arthritis. J Rheumatol. 2000;27(11):2611-6.

25. Antero DC, Parra AG, Miyazaki FH, Gehlen M, Skare TL. Secondary Sjogren's syndrome and disease activity of rheumatoid arthritis. Rev Assoc Med Bras. 2011;57(3):319-22.

26. Kosrirukvongs $P$, Ngowyutagon $P$, Pusuwan $P$, Koolvisoot $A$, Nilganuwong $S$. Prevalence of dry eye syndrome and Sjogren's syndrome in patients with rheumatoid arthritis. J Med Assoc Thai. 2012;95 Suppl 4:S61-9.

27. He J, Ding Y, Feng M, Guo J, Sun X, Zhao J, et al. Characteristics of Sjogren's syndrome in rheumatoid arthritis. Rheumatology (Oxford). 2013;52(6):1084-9.

28. Abdelghani KB, Mahmoud I, Chatelus E, Sordet C, Gottenberg JE, Sibilia J. Clinical and Serological Features of SjogrenSyndrome in Patients with Rheumatoid Arthritis. British Journal of Medicine \& Medical Research. 2016;5(10):1287-93.

29. Young A, Koduri G. Extra-articular manifestations and complications of rheumatoid arthritis. Best Pract Res Clin Rheumatol. 2007;21(5):907-27.

30. Fujita M, Igarashi T, Kurai T, Sakane M, Yoshino S, Takahashi H. Correlation between dry eye and rheumatoid arthritis activity. Am J Ophthalmol. 2005;140(5):808-13.

31. Martinez Castro E, Olive Marques A, Bonet Llorach M, Carbonell Abello J, Cobo Valeri E, Junca Valdor S. [Rheumatoid arthritis and Sjogren's syndrome. Special reference to the course time of rheumatoid arthritis]. Med Clin (Barc). 1990;94(17):655-9.

32. Aliko A, Ciancaglini R, Alushi A, Tafaj A. Sicca symptoms, and lacrimal and salivary flow in Albanian patients with rheumatoid arthritis. J Oral Pathol Med. 2010;39(8):651-6.

33. Cimmino MA, Salvarani C, Macchioni P, Montecucco C, Fossaluzza V, Mascia MT, et al. Extra-articular manifestations in 587 Italian patients with rheumatoid arthritis. Rheumatol Int. 2000;19(6):213-7.

34. Prabu A, Marshall T, Gordon C, Plant T, Bawendi A, Heaton S, et al. Use of patient age and anti-Ro/La antibody status to determine the probability of patients with systemic lupus erythematosus and sicca symptoms fulfilling criteria for secondary Sjogren's syndrome. Rheumatology. 2003;42(1):189-91.

35. McDonagh JE, Isenberg DA. Development of additional autoimmune diseases in a population of patients with systemic lupus erythematosus. Ann Rheum Dis. 2000;59(3):230-2.

36. Manoussakis MN, Georgopoulou C, Zintzaras E, Spyropoulou M, Stavropoulou A, Skopouli FN, et al. Sjogren's syndrome associated with systemic lupus erythematosus: clinical and laboratory profiles and comparison with primary Sjogren's syndrome. Arthritis Rheum. 2004;50(3):882-91. 
37. Gilboe IM, Kvien TK, Uhlig T, Husby G. Sicca symptoms and secondary Sjogren's syndrome in systemic lupus erythematosus: comparison with rheumatoid arthritis and correlation with disease variables. Annals of the rheumatic diseases. 2001;60(12):1103-9.

38. Alonso MD, Martinez-Vazquez F, Riancho-Zarrabeitia L, Diaz de Teran T, Miranda-Filloy JA, Blanco R, et al. Sex differences in patients with systemic lupus erythematosus from Northwest Spain. Rheumatol Int. 2014;34(1):11-24.

39. Pan HF, Ye DQ, Wang Q, Li WX, Zhang N, Li XP, et al. Clinical and laboratory profiles of systemic lupus erythematosus associated with Sjogren syndrome in China: a study of 542 patients. Clin Rheumatol. 2008;27(3):339-43.

40. Lockshin MD, Levine $A B$, Erkan D. Patients with overlap autoimmune disease differ from those with 'pure' disease. Lupus Sci Med. 2015;2(1):e000084.

41. Aggarwal R, Anaya JM, Koelsch KA, Kurien BT, Scofield RH. Association between Secondary and Primary Sjogren's Syndrome in a Large Collection of Lupus Families. Autoimmune diseases. 2015;2015:298506.

42. Szanto A, Szodoray P, Kiss E, Kapitany A, Szegedi G, Zeher M. Clinical, serologic, and genetic profiles of patients with associated Sjogren's syndrome and systemic lupus erythematosus. Hum Immunol. 2006;67(11):924-30.

43. Koskenmies S, Jarvinen TM, Onkamo P, Panelius J, Tuovinen U, Hasan T, et al. Clinical and laboratory characteristics of Finnish lupus erythematosus patients with cutaneous manifestations. Lupus. 2008;17(4):337-47.

44. Baer AN, Maynard JW, Shaikh F, Magder LS, Petri M. Secondary Sjogren's syndrome in systemic lupus erythematosus defines a distinct disease subset. J Rheumatol. 2010;37(6):1143-9.

45. Nossent JC, Swaak AJ. Systemic lupus erythematosus VII: frequency and impact of secondary Sjogren's syndrome. Lupus. 1998;7(4):231-4.

46. Scofield RH, Bruner GR, Harley JB, Namjou B. Autoimmune thyroid disease is associated with a diagnosis of secondary Sjogren's syndrome in familial systemic lupus. Annals of the rheumatic diseases. 2007;66(3):410-3.

47. Osial TA, Jr., Whiteside TL, Buckingham RB, Singh G, Barnes EL, Pierce JM, et al. Clinical and serologic study of Sjogren's syndrome in patients with progressive systemic sclerosis. Arthritis and rheumatism. 1983;26(4):500-8.

48. Drosos AA, Andonopoulos AP, Costopoulos JS, Stavropoulos ED, Papadimitriou CS, Moutsopoulos HM. Sjogren's syndrome in progressive systemic sclerosis. The Journal of rheumatology. 1988;15(6):965-8.

49. Drosos AA, Pennec YL, Elisaf M, Lamour A, Acritidis NC, Jouquan JR, et al. Sjogren's syndrome in patients with the CREST variant of progressive systemic scleroderma. J Rheumatol. 1991;18(11):1685-8.

50. Avouac J, Sordet C, Depinay C, Ardizonne M, Vacher-Lavenu MC, Sibilia J, et al. Systemic sclerosis-associated Sjogren's syndrome and relationship to the limited cutaneous subtype: results of a prospective study of sicca syndrome in 133 consecutive patients. Arthritis and rheumatism. 2006;54(7):2243-9.

51. Salliot C, Mouthon L, Ardizzone M, Sibilia J, Guillevin L, Gottenberg JE, et al. Sjogren's syndrome is associated with and not secondary to systemic sclerosis. Rheumatology. 2007;46(2):321-6.

52. Kobak S, Oksel F, Aksu K, Kabasakal Y. The frequency of sicca symptoms and Sjogren's syndrome in patients with systemic sclerosis. International journal of rheumatic diseases. 2013;16(1):88-92.

53. Rojana-udomsart A, Needham M, Luo YB, Fabian V, Walters S, Zilko PJ, et al. The association of sporadic inclusion body myositis and Sjogren's syndrome in carriers of HLA-DR3 and the 8.1 MHC ancestral haplotype. Clin Neurol Neurosurg. 2011;113(7):559-63. 
54. Vancsa A, Gergely L, Ponyi A, Lakos G, Nemeth J, Szodoray P, et al. Myositis-specific and myositis-associated antibodies in overlap myositis in comparison to primary dermatopolymyositis: Relevance for clinical classification: retrospective study of 169 patients. Joint Bone Spine. 2010;77(2):125-30.

55. Dobloug GC, Antal EA, Sveberg L, Garen T, Bitter H, Stjarne J, et al. High prevalence of inclusion body myositis in Norway; a population-based clinical epidemiology study. Eur J Neurol. 2015;22(4):672-e41.

56. Kauppi M, Pukkala E, Isomaki H. Elevated incidence of hematologic malignancies in patients with Sjogren's syndrome compared with patients with rheumatoid arthritis (Finland). Cancer Causes Control. 1997;8(2):201-4.

57. Baldini C, Mosca M, Della Rossa A, Pepe P, Notarstefano C, Ferro F, et al. Overlap of ACA-positive systemic sclerosis and Sjogren's syndrome: a distinct clinical entity with mild organ involvement but at high risk of lymphoma. Clinical and experimental rheumatology. 2013;31(2):272-80.

58. Koszarny A, Majdan M, Dryglewska M, Tabarkiewicz J. Prevalence of selected organ-specific autoantibodies in rheumatoid arthritis and primary Sjogren's syndrome patients. Reumatologia. 2015;53(2):61-8.

59. Hernandez-Molina G, Avila-Casado C, Cardenas-Velazquez F, Hernandez-Hernandez C, Calderillo ML, Marroquin V, et al. Similarities and differences between primary and secondary Sjogren's syndrome. The Journal of rheumatology. 2010;37(4):800-8.

60. Streckfus C, Bigler L, Navazesh M, Al-Hashimi I. Cytokine concentrations in stimulated whole saliva among patients with primary Sjogren's syndrome, secondary Sjogren's syndrome, and patients with primary Sjogren's syndrome receiving varying doses of interferon for symptomatic treatment of the condition: a preliminary study. Clinical oral investigations. 2001;5(2):133-5.

61. Tishler M, Aharon A, Ehrenfeld M, Avni I, Bendet E, Bombardieri S, et al. Sjogren's syndrome in Israel: primary versus secondary disease. Clinical rheumatology. 1994;13(3):438-41.

62. Brown LE, Frits ML, lannaccone CK, Weinblatt ME, Shadick NA, Liao KP. Clinical characteristics of RA patients with secondary SS and association with joint damage. Rheumatology. 2015;54(5):816-20.

63. Andonopoulos AP, Skopouli FN, Dimou GS, Drosos AA, Moutsopoulos HM. Sjogren's syndrome in systemic lupus erythematosus. J Rheumatol. 1990;17(2):201-4.

\section{Figure legends:}

Figure 1: Flowchart of study selection.

Figure 2: Meta-analysis of the prevalence of sSS in SLE patients.

Figure 3: Meta-analysis of the prevalence of sSS in RA patients. 
Table 1: Studies reporting the disease duration and relationship with the sSS prevalence in RA patients.

\begin{tabular}{lll}
\hline Study & Disease duration & $\begin{array}{l}\text { Relation between } \\
\text { prevalence of sSS and RA } \\
\text { disease duration }\end{array}$ \\
\hline Uhlig et al.,1999 & 12.2 years & None \\
\hline Antero et al., 2011 & $10.2+/-7$ years & None \\
Abdelghani, 2014 & 15.6 years & None \\
Haga et al.,2012 & 10.63 years & None \\
Carmona et al.,2003 & - & Prevalence at 10 years $17 \%$, \\
& & and $25 \%$ after 30 years \\
Young et al.,2000 & 10 years & $\begin{array}{l}\text { Prevalence at } 1 \text { year } 4 \%, \text { and } \\
12 \% \text { after } 10 \text { years }\end{array}$ \\
\end{tabular}


Table 2: Studies included in the systematic review

\begin{tabular}{|c|c|c|c|c|c|c|c|c|c|c|}
\hline & $\begin{array}{l}\text { Study } \\
\text { design }\end{array}$ & Country & $\begin{array}{l}\text { Number } \\
\text { of } \\
\text { patients }\end{array}$ & $\begin{array}{l}\text { Criteria } \\
\text { used }\end{array}$ & $\begin{array}{l}\text { Number } \\
\text { of sSS } \\
\text { cases (\%) }\end{array}$ & Ethnicity & $\begin{array}{l}\text { Female/male } \\
\text { (n) }\end{array}$ & $\begin{array}{l}\text { Disease duration } \\
\text { (years/months) }\end{array}$ & $\begin{array}{l}\text { Salivary gland } \\
\text { biopsy }\end{array}$ & Study quality \\
\hline & & & & & \multicolumn{6}{|c|}{ Systemic lupus erythematosus } \\
\hline $\begin{array}{l}\text { Nossent et al, } \\
1998\end{array}$ & $\mathrm{P}$ & $\begin{array}{l}\text { Norway/ } \\
\text { Netherlands }\end{array}$ & 138 & ECC & $\begin{array}{l}N=27 \\
(19.6 \%)\end{array}$ & NA & $22 / 5$ & 8 years & $\begin{array}{ll}12 & \text { patients } \\
\text { tested } & \end{array}$ & Moderate \\
\hline $\begin{array}{l}\text { McDonagh et al, } \\
2000\end{array}$ & $\mathrm{R}$ & $\begin{array}{l}\text { UK } \\
\text { (London) }\end{array}$ & 215 & ECC & $\begin{array}{l}N=28 \\
(13 \%)\end{array}$ & NA & NA & 18 years & All tested & Moderate \\
\hline $\begin{array}{l}\text { Gilboe et al, } \\
2001\end{array}$ & $\mathrm{R}$ & $\begin{array}{l}\text { Norway } \\
\text { (Oslo) }\end{array}$ & 81 & ECC & $\begin{array}{l}N=9 \\
(11 \%)\end{array}$ & Caucasian & $9 / 0$ & 8 years & NA & Moderate \\
\hline $\begin{array}{l}\text { Bowman et al, } \\
2003\end{array}$ & CS & $\begin{array}{l}\text { UK } \\
\text { (Birmingham) }\end{array}$ & 96 & AECG & $\begin{array}{l}N=18 \\
(19 \%)\end{array}$ & Caucasian & $18 / 0$ & 10.9 years & NA & Moderate \\
\hline $\begin{array}{l}\text { Manoussakis et } \\
\text { al, } 2004\end{array}$ & $\mathrm{R}$ & $\begin{array}{l}\text { Greece } \\
\text { (Athens) }\end{array}$ & 283 & AECG & $\begin{array}{l}N=26 \\
(9 \%)\end{array}$ & Caucasian & $26 / 0$ & 3.5 years & All tested & Good \\
\hline $\begin{array}{l}\text { Szanto et al, } \\
2006\end{array}$ & $\mathrm{R}$ & $\begin{array}{l}\text { Hungary } \\
\text { (Debrecen) }\end{array}$ & 362 & AECG & $\begin{array}{l}N=56 \\
(15 \%)\end{array}$ & Caucasian & $52 / 4$ & 8.1 years & All tested & Good \\
\hline $\begin{array}{l}\text { Scofield et al, } \\
2007\end{array}$ & $\mathrm{R}$ & $\begin{array}{l}\text { USA } \\
\text { (Oklahoma) }\end{array}$ & 1138 & NA & $\begin{array}{l}N=169 \\
(15 \%)\end{array}$ & NA & NA & NA & NA & Poor \\
\hline
\end{tabular}




\begin{tabular}{|c|c|c|c|c|c|c|c|c|c|c|}
\hline Pan et al, 2008 & $\mathrm{R}$ & China & 542 & AECG & $\begin{array}{l}N=35 \\
(6 \%)\end{array}$ & Asian Chinese & $32 / 3$ & 3.7 years & All tested & Good \\
\hline $\begin{array}{l}\text { Koskenmies et } \\
\text { al, } 2008\end{array}$ & $\mathrm{R}$ & $\begin{array}{l}\text { Finland } \\
\text { (Helsinki) }\end{array}$ & 77 & NA & $\begin{array}{l}N=17 \\
(22 \%)\end{array}$ & NA & NA & NA & NA & Poor \\
\hline $\begin{array}{l}\text { Baer et al, } \\
2010\end{array}$ & CS & $\begin{array}{l}\text { USA } \\
\text { (Marylands) }\end{array}$ & 1790 & AECG & $\begin{array}{l}N=259 \\
(14.5 \%)\end{array}$ & $\begin{array}{l}\text { White }=70.7 \\
\text { African } \\
\text { American= } 25.5 \\
\text { Hispanic= } 1.9 \\
\text { Asian= } 1.2\end{array}$ & $253 / 6$ & 19.5 years & All tested & Good \\
\hline Maria et al, 2013 & $\mathrm{CS} / \mathrm{R}$ & $\begin{array}{l}\text { Spain } \\
\text { (Lugo) }\end{array}$ & 150 & NA & $\begin{array}{l}N=27 \\
(18 \%)\end{array}$ & NA & $27 / 0$ & NA & NA & Poor \\
\hline $\begin{array}{l}\text { Lockshin et al, } \\
2015\end{array}$ & $\mathrm{R}$ & USA & 600 & Other & $\begin{array}{l}N=28 \\
(5 \%)\end{array}$ & NA & NA & NA & NA & Poor \\
\hline $\begin{array}{l}\text { Aggarwal et al, } \\
2015\end{array}$ & L & USA & 2694 & AECG & $\begin{array}{l}N=548 \\
(20 \%)\end{array}$ & $\begin{array}{l}\text { Mixed (White, } \\
\text { Black, } \\
\text { Hispanic, } \\
\text { Asian and } \\
\text { native) }\end{array}$ & $504 / 44$ & 20 years & NA & Good \\
\hline \multicolumn{11}{|c|}{ Rheumatoid arthritis } \\
\hline $\begin{array}{l}\text { Andonopoulos et } \\
\text { al, } 1987\end{array}$ & $P$ & Greece & 111 & Other & $\begin{array}{l}N=34 \\
(31 \%)\end{array}$ & Greek & NA & 3.3 - 9.1 years & All tested & Good \\
\hline $\begin{array}{l}\text { Martinez Castro } \\
\text { et al, } 1990\end{array}$ & CS & Spain & 45 & NA & $\begin{array}{l}N=24 \\
(55 \%)\end{array}$ & Spanish & NA & NA & All tested & Poor \\
\hline
\end{tabular}




\begin{tabular}{|c|c|c|c|c|c|c|c|c|c|c|}
\hline $\begin{array}{l}\text { Drosos et al, } \\
1992\end{array}$ & CS & $\begin{array}{l}\text { Greece/British } \\
\text { loannina/London }\end{array}$ & $\begin{array}{l}G=108 \\
B=107\end{array}$ & Other & $\begin{array}{l}\text { GK N=43 } \\
(39.8 \%) \\
\text { BS N=17 } \\
(15.9 \%)\end{array}$ & Greek/British & NA & NA & All tested & Moderate \\
\hline Uhlig et al, 1999 & CS & $\begin{array}{l}\text { Norway } \\
\text { (Oslo) }\end{array}$ & 636 & ECCN & $\begin{array}{l}N=46 \\
(7 \%)\end{array}$ & NA & NA & 12.2 years & NA & Moderate \\
\hline $\begin{array}{l}\text { Cimmino et al, } \\
2000\end{array}$ & CS & $\begin{array}{l}\text { Italy } \\
\text { (Northern) }\end{array}$ & 587 & Other & $\begin{array}{l}N=103 \\
(17.5 \%)\end{array}$ & Italian & NA & 10 years & NA & Poor \\
\hline $\begin{array}{l}\text { Mattey et al, } \\
2000\end{array}$ & CS & $\begin{array}{l}\text { Spain } \\
\text { (Lugo) }\end{array}$ & 179 & Other & $\begin{array}{l}N=22 \\
(12.3 \%)\end{array}$ & NA & NA & NA & NA & Poor \\
\hline $\begin{array}{l}\text { Young et al, } \\
2000\end{array}$ & $P$ & UK & 732 & NA & $\begin{array}{l}N=54 \\
(7 \%)\end{array}$ & NA & $46 / 9$ & $4-11$ months & NA & Poor \\
\hline $\begin{array}{l}\text { loannidis et al, } \\
2002\end{array}$ & CS & $\begin{array}{l}\text { Greece } \\
\text { (loannina/ } \\
\text { Athens) }\end{array}$ & 174 & ECC & $\begin{array}{l}N=57 \\
(32.7 \%)\end{array}$ & Greek & NA & 10.5 years & NA & Moderate \\
\hline $\begin{array}{l}\text { Turesson et al, } \\
2003\end{array}$ & $\mathrm{R}$ & $\begin{array}{l}\text { USA } \\
\text { (Minnesota) }\end{array}$ & 609 & Other & $\begin{array}{l}N=58 \\
(9.5 \%)\end{array}$ & NA & NA & 46 years & NA & Poor \\
\hline $\begin{array}{l}\text { Carmona et al, } \\
2003\end{array}$ & CS & Spain & 788 & ECC & $\begin{array}{l}N=134 \\
(17 \%)\end{array}$ & Spanish & NA & NA & NA & Moderate \\
\hline Fujita et al, 2005 & $P$ & $\begin{array}{l}\text { Japan } \\
\text { (Tokyo) }\end{array}$ & 72 & Japanese & $\begin{array}{l}N=7 \\
(10 \%)\end{array}$ & Japanese & $7 / 0$ & NA & NA & Poor \\
\hline $\begin{array}{l}\text { Calgüneri et al, } \\
2006\end{array}$ & $\mathrm{R}$ & $\begin{array}{l}\text { Turkey } \\
\text { (Ankara) }\end{array}$ & 526 & NA & $\begin{array}{l}N=28 \\
(5.3 \%)\end{array}$ & NA & NA & NA & NA & Poor \\
\hline $\begin{array}{l}\text { Antero et al, } \\
2011\end{array}$ & CS & $\begin{array}{l}\text { Brazil } \\
\text { (Curitiba) }\end{array}$ & 82 & AECG & $\begin{array}{l}N=20 \\
(24.3 \%)\end{array}$ & NA & $18 / 2$ & 10.2 years & All tested & Good \\
\hline
\end{tabular}




\begin{tabular}{|c|c|c|c|c|c|c|c|c|c|c|}
\hline $\begin{array}{l}\text { Kosrirukvongs et } \\
\text { al, } 2012\end{array}$ & CS & $\begin{array}{l}\text { Thailand } \\
\text { (Siriraj) }\end{array}$ & 61 & Other & $\begin{array}{l}N=14 \\
(22.2 \%)\end{array}$ & NA & NA & NA & NA & Poor \\
\hline Haga et al, 2012 & CS & $\begin{array}{l}\text { Denmark } \\
\text { (Esbjerg) }\end{array}$ & 307 & AECG & $\begin{array}{l}N=11 \\
(3.6 \%)\end{array}$ & NA & NA & 10.6 years & NA & Moderate \\
\hline Aliko et al, 2010 & CS & $\begin{array}{l}\text { Albania } \\
\text { (Tirana) }\end{array}$ & 88 & ECC & $\begin{array}{l}\mathrm{N}=13 \\
(14.8 \%)\end{array}$ & Albanian & NA & 9.5 years & NA & Moderate \\
\hline He J et al, 2013 & $\mathrm{R}$ & $\begin{array}{l}\text { China } \\
\text { (Beijing) }\end{array}$ & 509 & AECG & $\begin{array}{l}N=74 \\
(14.5 \%)\end{array}$ & Chinese & $64 / 10$ & 15.10 months & $\begin{array}{ll}16 & \text { patients } \\
\text { tested } & \end{array}$ & Good \\
\hline $\begin{array}{l}\text { Abdelghani, } \\
2014\end{array}$ & CS & $\begin{array}{l}\text { France } \\
\text { (Strasbourg) }\end{array}$ & 76 & AECG & $\begin{array}{l}N=11 \\
(14 \%)\end{array}$ & French & NA & 15.6 years & 9 patients tested & Moderate \\
\hline $\begin{array}{l}\text { Brown et al., } \\
2015\end{array}$ & CS & $\begin{array}{l}\text { USA } \\
\text { (Boston) }\end{array}$ & 829 & AECG & $\begin{array}{l}N=85 \\
(10.3 \%)\end{array}$ & White $=89.4$ & $76 / 9$ & 16.9 years & NA & Good \\
\hline \multicolumn{11}{|c|}{ Systemic sclerosis } \\
\hline Osial et al, 1983 & CS & $\begin{array}{l}\text { USA } \\
\text { (Pennsylvania) }\end{array}$ & 58 & Other & $\begin{array}{l}N=17 \\
(29 \%)\end{array}$ & NA & $16 / 1$ & 7.3 years & All tested & Good \\
\hline $\begin{array}{l}\text { Andonopoulos et } \\
\text { al, } 1988\end{array}$ & $P$ & Greece & 44 & Other & $\begin{array}{l}N=9 \\
(20.5 \%)\end{array}$ & NA & NA & NA & All tested & Poor \\
\hline $\begin{array}{l}\text { Drosos et al, } \\
1991\end{array}$ & CS & Greece & 23 & NA & $\begin{array}{l}N=14 \\
(60 \%)\end{array}$ & NA & NA & NA & All tested & Poor \\
\hline $\begin{array}{l}\text { Avouac et al, } \\
2006\end{array}$ & $P$ & $\begin{array}{l}\text { France } \\
\text { (Paris) }\end{array}$ & 133 & AECG & $\begin{array}{l}N=19 \\
(14 \%)\end{array}$ & NA & $16 / 3$ & 7 years & $\begin{array}{ll}91 & \text { patients } \\
\text { tested } & \end{array}$ & Good \\
\hline
\end{tabular}




\begin{tabular}{|c|c|c|c|c|c|c|c|c|c|c|}
\hline Salliot et al, 2006 & $\mathrm{R}$ & $\begin{array}{l}\text { France } \\
\text { (Paris) }\end{array}$ & 121 & AECG & $\begin{array}{l}N=27 \\
(22 \%)\end{array}$ & NA & $24 / 3$ & 7.3 years & All tested & Good \\
\hline Kobak et al, 2013 & CS & $\begin{array}{l}\text { Turkey } \\
\text { (Izmir) }\end{array}$ & 118 & AECG & $\begin{array}{l}N=40 \\
(33.9 \%)\end{array}$ & NA & $38 / 2$ & 8.2 years & $\begin{array}{ll}74 & \text { patients } \\
\text { tested } & \end{array}$ & Good \\
\hline $\begin{array}{l}\text { Baldini et al., } \\
2013\end{array}$ & $\mathrm{R}$ & Italy (Pisa) & $\begin{array}{l}209- \\
\text { systemic } \\
\text { sclerosis } \\
402 \\
\text { pSS }\end{array}$ & $\begin{array}{l}\text { Le Roy } \\
\text { AECG }\end{array}$ & $\mathrm{N}=41$ & NA & NA & NA & NA & Moderate \\
\hline \multicolumn{11}{|c|}{ Myositis } \\
\hline $\begin{array}{l}\text { Rojana- } \\
\text { Udomsart et al, } \\
2011\end{array}$ & CS & Australia & 6 & AECG & $\begin{array}{l}N=6 \\
(12 \%)\end{array}$ & NA & $5 / 1$ & 20 years & NA & Poor \\
\hline $\begin{array}{l}\text { Vancsa et al, } \\
2010\end{array}$ & $\mathrm{CS} / \mathrm{R}$ & Hungary & 169 & NA & $\begin{array}{l}N=9 \\
(23 \%)\end{array}$ & NA & NA & 6 years & NA & Poor \\
\hline $\begin{array}{l}\text { Dobloug et al, } \\
2014\end{array}$ & CS & Norway & 100 & NA & $\begin{array}{l}N=10 \\
(10 \%)\end{array}$ & NA & NA & 5.5 years & NA & Poor \\
\hline
\end{tabular}

Legend: CS - cross-sectional study; NA - information not available; P - prospective study; R - retrospective study. 


\begin{tabular}{|c|c|c|c|c|c|c|c|c|c|c|c|c|}
\hline Disease/Ref & $\begin{array}{l}\text { Arthritis } \\
(\% \\
\text { patients })\end{array}$ & $\begin{array}{l}\text { Swollen } \\
\text { joints } \\
\text { (\% } \\
\text { patients) }\end{array}$ & $\begin{array}{l}\text { Tender } \\
\text { joints } \\
\text { (\% } \\
\text { patients) }\end{array}$ & $\begin{array}{l}\text { DAS } 28 \\
\text { score }\end{array}$ & $\begin{array}{l}\text { Raynaud' } \\
\text { s } \\
\text { (\% } \\
\text { patients) }\end{array}$ & $\begin{array}{l}\text { Photo } \\
\text { sensitivity } \\
\text { (\% } \\
\text { patients) }\end{array}$ & $\begin{array}{l}\text { Lymph } \\
\text { adenopathy } \\
\text { (\% patients) }\end{array}$ & $\begin{array}{l}\text { Thyroiditis } \\
\text { (\% } \\
\text { patients) }\end{array}$ & $\begin{array}{l}\text { Lung } \\
\text { involvement } \\
\text { (\% } \\
\text { patients) }\end{array}$ & $\begin{array}{l}\text { Renal } \\
\text { involvement } \\
\text { (\% } \\
\text { patients) }\end{array}$ & $\begin{array}{l}\text { Nervous } \\
\text { system } \\
\text { involvement } \\
\text { (\% patients) }\end{array}$ & $\begin{array}{l}\text { Lymphoma } \\
\text { (\% patients) }\end{array}$ \\
\hline \multicolumn{13}{|c|}{ SLE vs. SLE-sSS patients } \\
\hline $\begin{array}{l}\text { Nossent et al, } \\
1998\end{array}$ & $\begin{array}{l}88 \text { vs. } 92 \\
(p=N S)\end{array}$ & & & & & & & & & $\begin{array}{l}38 \text { vs. } 19 \\
(\mathbf{p}=\mathbf{0 . 0 4})\end{array}$ & $\begin{array}{l}\text { NPSLE } \\
18 \text { vs. } 19 \\
(p=N S)\end{array}$ & $0 \%$ \\
\hline $\begin{array}{l}\text { Gilboe et al, } \\
2001\end{array}$ & & & & & & & & & & $\begin{array}{l}19 \text { vs. } 0 \\
(\mathbf{p}<\mathbf{0 . 0 5})\end{array}$ & & \\
\hline $\begin{array}{l}\text { Manoussakis et } \\
\text { al, } 2004\end{array}$ & $\begin{array}{l}51.3 \text { vs. } \\
76.9 \\
(p=0.27)\end{array}$ & & & & $\begin{array}{l}43.4 \text { vs. } \\
80.8 \\
(\mathbf{p}<\mathbf{0 . 0 0 1})\end{array}$ & & $\begin{array}{c}46.1 \text { vs. } 19.2 \\
(\mathbf{p}=\mathbf{0 . 0 0 4})\end{array}$ & & $\begin{array}{l}11.8 \text { vs. } 11.5 \\
(p=0.891)\end{array}$ & $\begin{array}{l}55.3 \text { vs. } 11.5 \\
(\mathbf{p}=\mathbf{0 . 0 0 5})\end{array}$ & $\begin{array}{l}19.7 \text { vs. } 11.5 \\
(p=0.55)\end{array}$ & $0 \%$ \\
\hline $\begin{array}{l}\text { Szanto et al, } \\
2006\end{array}$ & & & & & $\begin{array}{l}28 \text { vs. } 35.7 \\
(p=0.396)\end{array}$ & & & $\begin{array}{l}6 \text { vs. } 21.4 \\
(p=0.023)\end{array}$ & $\begin{array}{l}24 \text { vs. } 28.5 \\
(p=0.59)\end{array}$ & $\begin{array}{l}66 \text { vs. } 57.1 \\
(p=0.312)\end{array}$ & $\begin{array}{l}36 \text { vs. } 25 \\
(p=0.21)\end{array}$ & \\
\hline $\begin{array}{l}\text { Scofield et al, } \\
2007\end{array}$ & & & & & & & & $\begin{array}{l}12.7 \text { vs. } 29.6 \\
(\mathbf{p}<\mathbf{0 . 0 0 0})\end{array}$ & & & & \\
\hline Pan et al, 2008 & & & $\begin{array}{l}60.9 \text { vs. } \\
77.1 \\
(p=0.056)\end{array}$ & & & $\begin{array}{l}14.2 \text { vs. } \\
8.6 \\
(p=0.45)\end{array}$ & & & & $\begin{array}{l}66.7 \text { vs. } 48.6 \\
(\mathbf{p}=\mathbf{0 . 0 3})\end{array}$ & $\begin{array}{l}9.5 \text { vs. } 2.9 \\
(p=0.35)\end{array}$ & \\
\hline $\begin{array}{l}\text { Baer et al, } \\
2010\end{array}$ & $\begin{array}{l}73.2 \text { vs. } \\
81.3 \\
(p=0.006)\end{array}$ & & & & $\begin{array}{l}49.5 \text { vs. } 66 \\
(\mathbf{p}<\mathbf{0 . 0 0 1})\end{array}$ & $\begin{array}{l}52.9 \text { vs. } \\
68.3 \\
(\mathbf{p}=\mathbf{0 . 0 0 1})\end{array}$ & & & & $\begin{array}{l}\text { Proteinuria } \\
43.1 \text { vs. } 29.0 \\
(\mathbf{p}<\mathbf{0 . 0 0 1 )} \\
\text { Haematuria } \\
30.9 \text { vs. } 22.8 \\
(\mathbf{p}=\mathbf{0 . 0 0 8}) \\
\text { Nephrotic } \\
\text { syndrome } \\
20 \text { vs. } 8.9 \\
(\mathbf{p}<\mathbf{0 . 0 0 1})\end{array}$ & $\begin{array}{l}\text { Psychosis } \\
3 \text { vs. } 6.6 \\
\text { (p=0.005) } \\
\text { Seizures } \\
9.3 \text { vs. } 11.6 \\
(p=0.25)\end{array}$ & \\
\hline
\end{tabular}




\begin{tabular}{|c|c|c|c|c|c|c|c|c|c|c|c|}
\hline \multicolumn{12}{|c|}{ RA vs. RA-sSS patients } \\
\hline $\begin{array}{l}\text { Uhlig } \\
1999\end{array}$ & al & 100 & $\begin{array}{l}7.0 \text { vs. } \\
8.5 \\
(p=0.17)\end{array}$ & $\begin{array}{l}6.1 \text { vs. } 9.6 \\
(\mathbf{p}=<\mathbf{0 . 0 1})\end{array}$ & $\begin{array}{l}4.20 \text { vs. } \\
5.08 \\
(\mathbf{p}<\mathbf{0 . 0 0 1 )}\end{array}$ & & & & & & \\
\hline $\begin{array}{l}\text { Antero } \\
2011\end{array}$ & t al & 100 & & & $\begin{array}{l}3.35 \text { vs. } \\
2.81 \\
(p=0.1)\end{array}$ & & & & & & \\
\hline $\begin{array}{l}\text { Haga } \\
2012\end{array}$ & al & 100 & $\begin{array}{l}0.28 \text { vs. } \\
0.73 \\
(p=N S)\end{array}$ & $\begin{array}{l}1.1 \text { vs. } 2.2 \\
(p=N S)\end{array}$ & $\begin{array}{l}3.1 \text { vs. } 2.7 \\
(p=N S)\end{array}$ & & & & & & \\
\hline He J et al & 2013 & 100 & $\begin{array}{l}12.9 \text { vs. } \\
15.8 \\
(\mathbf{p}<\mathbf{0 . 0 5})\end{array}$ & $\begin{array}{l}12.1 \text { vs. } \\
14.5 \\
(\mathbf{p}=\mathbf{0 . 0 1 9})\end{array}$ & $\begin{array}{l}5.9 \text { vs. } 6.4 \\
(\mathbf{p}=\mathbf{0 . 0 0 9})\end{array}$ & & $\begin{array}{l}7.59 \text { vs. } 10.8 \\
(\mathrm{p}=0.346)\end{array}$ & $\begin{array}{l}27.1 \text { vs. } 21.6 \\
(\mathrm{p}=0.320)\end{array}$ & $\begin{array}{l}11.7 \text { vs. } 44.6 \\
(\mathbf{p}<\mathbf{0 . 0 0 1})\end{array}$ & $\begin{array}{l}4.81 \text { vs. } 14.9 \\
(\mathbf{p}=\mathbf{0 . 0 0 2})\end{array}$ & $\begin{array}{l}0.23 \text { vs. } 2.7 \\
(\mathbf{p}=\mathbf{0 . 0 1 0})\end{array}$ \\
\hline $\begin{array}{l}\text { Abdelgha } \\
\text { al, } 2014\end{array}$ & ii e & 100 & $\begin{array}{l}3.7 \text { vs. } \\
3.2 \\
(p=0.4)\end{array}$ & $\begin{array}{l}6.2 \text { vs. } 5.6 \\
(p=0.4)\end{array}$ & $\begin{array}{l}4.13 \text { vs. } \\
4.05 \\
(p=0.8)\end{array}$ & $\begin{array}{l}1.5 \text { vs. } \\
27.2 \\
(\mathbf{p}=\mathbf{0 . 0 1})\end{array}$ & & & & & \\
\hline $\begin{array}{l}\text { Brown } \\
2015 \\
\end{array}$ & $\begin{array}{ll}t & a l . \\
\end{array}$ & 100 & & & $\begin{array}{l}4.3 \text { vs. } 3.2 \\
(\mathbf{p}=\mathbf{0 . 0 1})\end{array}$ & & & & & & \\
\hline \multicolumn{12}{|c|}{ SSc vs. SSc-sSS patients } \\
\hline Osial et a & 198 & & & & & & & & $\begin{array}{l}\text { Unspecified } \\
\text { pulmonary } \\
\text { disease } \\
64 \text { vs. } 65\end{array}$ & 9 vs. 12 & \\
\hline $\begin{array}{l}\text { Avouac } \\
2006\end{array}$ & et al & & & & & & & & $\begin{array}{l}\text { Lung } \\
\text { fibrosis } \\
45 \text { vs. } 11 \\
\text { (p=0.02) } \\
\text { PAH } \\
19 \text { vs. } 11 \\
\text { (NS) }\end{array}$ & & \\
\hline $\begin{array}{l}\text { Salliot } \\
2006\end{array}$ & $t \quad$ al & & & & & $\begin{array}{l}94.7 \text { vs. } \\
92.6 \\
(p=0.98)\end{array}$ & & & $\begin{array}{l}\text { Lung } \\
\text { fibrosis } \\
29 \text { vs. 11.1 } \\
(\mathbf{p}=\mathbf{0 . 0 5}) \\
\text { PAH 15.1 } \\
\text { vs. 7.4 } \\
(p=0.60)\end{array}$ & $\begin{array}{l}14.9 \text { vs. } 3.7 \\
(p=0.21)\end{array}$ & \\
\hline
\end{tabular}




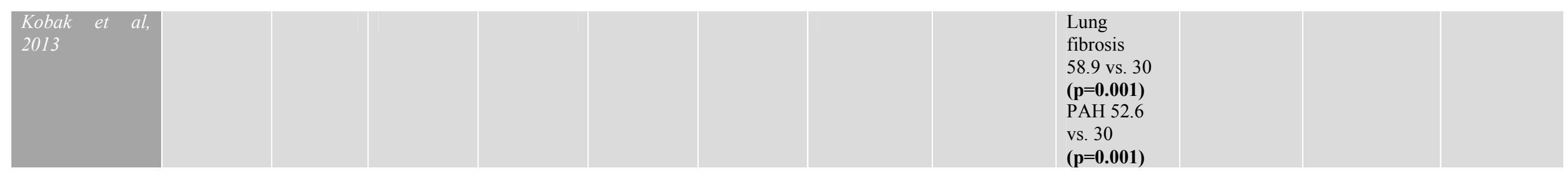

Legend: PAH - pulmonary arterial hypertension; NS - not significant; NPSLE- neuropsychiatric lupus.

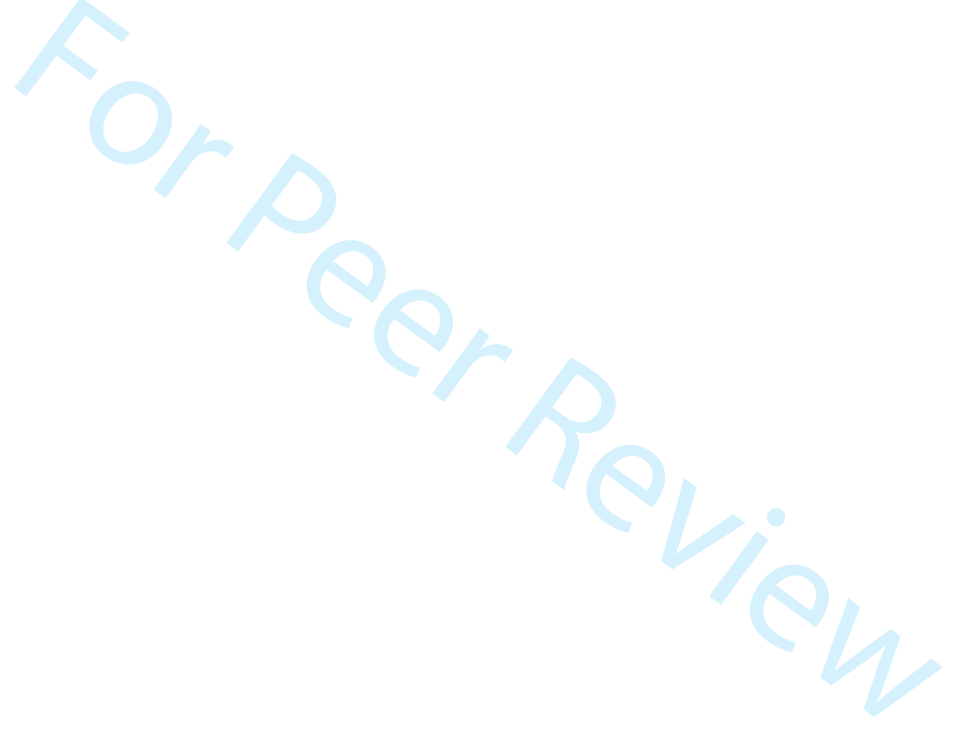




\begin{tabular}{|c|c|c|c|c|c|c|c|c|c|c|}
\hline Disease/Ref & $\begin{array}{l}\text { Ro/ SSA } \\
\text { antibodies } \\
\text { (\% } \\
\text { patients) }\end{array}$ & $\begin{array}{l}\text { La/ SSB } \\
\text { antibodies } \\
(\% \\
\text { patients) }\end{array}$ & $\begin{array}{l}\text { RF } \\
(\% \\
\text { patients/ } \\
\text { U/mL) }\end{array}$ & $\begin{array}{l}\text { ANA } \\
(\% \\
\text { patients })\end{array}$ & $\begin{array}{l}\text { dsDNA } \\
(\% \\
\text { patients })\end{array}$ & $\begin{array}{l}\text { Anti } \\
\text { CCP } \\
(\% \\
\text { patients })\end{array}$ & $\begin{array}{l}\text { Other antibodies/ } \\
\text { markers } \\
\text { (\% patients) }\end{array}$ & $\begin{array}{l}\text { ESR/CRP } \\
(\mathrm{mm} / \mathrm{h} ; \\
\mathrm{mg} / \mathrm{l})\end{array}$ & $\begin{array}{l}\text { Hyper } \\
\text { gamma } \\
\text { globulinaemia } \\
\text { (\% patients) }\end{array}$ & $\begin{array}{l}\text { Thrombo } \\
\text { Cytopaenia } \\
\text { (\% patients) }\end{array}$ \\
\hline \multicolumn{11}{|c|}{ SLE vs. SLE-sSS patients } \\
\hline $\begin{array}{l}\text { Nossent et } \\
\text { al, } 1998\end{array}$ & $\begin{array}{l}44 \text { vs. } 48 \\
(p=N S)\end{array}$ & $\begin{array}{l}38 \text { vs. } 33 \\
(p=N S)\end{array}$ & & $\begin{array}{l}88 \text { vs. } 87 \\
(p=N S)\end{array}$ & $\begin{array}{l}71 \text { vs. } 53 \\
(p=N S)\end{array}$ & & $\begin{array}{l}\text { Anticardiolipin } \\
33 \text { vs. } 41(\mathrm{p}=\mathrm{NS}) \\
\text { Anti-Sm } \\
21 \text { vs. } 15(\mathrm{p}=\mathrm{NS}) \\
\text { Anti-U1 nRNP } \\
26 \text { vs. } 25(\mathrm{p}=\mathrm{NS})\end{array}$ & & & $\begin{array}{l}9 \text { vs. } 26 \\
(\mathbf{p}<\mathbf{0 . 0 5})\end{array}$ \\
\hline $\begin{array}{l}\text { Uhlig et al, } \\
1999\end{array}$ & & & $\begin{array}{l}48.2 \mathrm{U} / \mathrm{mL} \\
\text { vs. } 62.2 \\
\mathrm{U} / \mathrm{mL} \\
(p=0.08)\end{array}$ & & & & & $\begin{array}{l}\text { ESR } \\
18.6 \text { vs. } 26.8 \\
(\mathbf{p}<\mathbf{0 . 0 1}) \\
\text { CRP } \\
11.9 \text { vs. } 13.8 \\
(p=0.33)\end{array}$ & & \\
\hline $\begin{array}{l}\text { Gilboe et al, } \\
2001\end{array}$ & $\begin{array}{l}36 \text { vs. } 89 \\
(\mathbf{p}=\mathbf{0 . 0 5})\end{array}$ & $\begin{array}{l}11 \text { vs. } 56 \\
(\mathbf{p}=\mathbf{0 . 0 5})\end{array}$ & & & $\begin{array}{l}60 \text { vs. } 44 \\
(\mathbf{p}=\mathbf{0 . 0 5})\end{array}$ & & & & & \\
\hline $\begin{array}{l}\text { Manoussakis } \\
\text { et al, } 2004\end{array}$ & $\begin{array}{l}23.9 \text { vs. } \\
38.5 \\
(\mathbf{p}=\mathbf{0 . 0 0 8})\end{array}$ & $\begin{array}{l}7.0 \text { vs. } \\
38.5 \\
(\mathbf{p}=<\mathbf{0 . 0 0 1} \\
)\end{array}$ & $\begin{array}{l}28.6 \text { vs. } 64 \\
(\mathbf{p}<\mathbf{0 . 0 0 1})\end{array}$ & $\begin{array}{l}100 \% \text { both } \\
\text { groups }\end{array}$ & $\begin{array}{l}77.3 \text { vs. } \\
69.2 \\
(p=0.436)\end{array}$ & & $\begin{array}{l}\text { Anticardiolipin antibodies } \\
52.9 \text { vs. } 45.8(\mathrm{p}=0.639) \\
\text { Anti-U1 nRNP antibodies } \\
12.7 \text { vs. } 11.5(\mathrm{p}=0.999) \\
\text { Anti-Sm } \\
11.3 \text { vs. } 7.7(\mathrm{p}=0.999) \\
\text { Cryoglobulins } \\
14.7 \text { vs. } 15.8(\mathrm{p}=0.999)\end{array}$ & & & $\begin{array}{l}26.3 \text { vs. } 7.7 \\
(\mathbf{p}=\mathbf{0 . 0 3})\end{array}$ \\
\hline $\begin{array}{l}\text { Szanto et al, } \\
2006\end{array}$ & $\begin{array}{l}74 \text { vs. } \\
94.64 \\
(\mathbf{p}<\mathbf{0 . 0 1})\end{array}$ & $\begin{array}{l}44 \text { vs. } \\
73.21 \\
(\mathbf{p}<\mathbf{0 . 0 1})\end{array}$ & $\begin{array}{l}31.65 \mathrm{U} / \mathrm{m} \\
\mathrm{L} \text { vs. } \\
120.39 \\
\mathrm{u} / \mathrm{mL} \\
(\mathrm{p}=0.126)\end{array}$ & & $\begin{array}{l}223.35 \text { vs. } \\
132.51 \\
(\mathbf{p}<\mathbf{0 . 0 1})\end{array}$ & & $\begin{array}{l}\text { Anti-U1 nRNP } \\
41.6 \text { vs. } 37.1(p=0.603) \\
\text { Anti-Sm } \\
38.7 \text { vs. } 22.9(p=0.06)\end{array}$ & & & $\begin{array}{l}36 \text { vs. } 25 \\
(p=0.218)\end{array}$ \\
\hline
\end{tabular}




\begin{tabular}{|c|c|c|c|c|c|c|c|c|c|c|}
\hline $\begin{array}{l}\text { Pan et al, } \\
2008\end{array}$ & $\begin{array}{l}27.6 \text { vs. } \\
71.4 \\
(\mathbf{p}<\mathbf{0 . 0 0 1})\end{array}$ & $\begin{array}{l}17.4 \text { vs. } \\
51.4 \\
(\mathbf{p}<\mathbf{0 . 0 0 1 )}\end{array}$ & & $\begin{array}{l}74.4 \text { vs. } \\
85.7 \\
(p=0.13)\end{array}$ & $\begin{array}{l}38.3 \text { vs. } 60 \\
(\mathbf{p}=\mathbf{0 . 0 1 1})\end{array}$ & & & & & $\begin{array}{l}42 \text { vs. } 40 \\
(p=0.816)\end{array}$ \\
\hline $\begin{array}{l}\text { Baer et al, } \\
2010\end{array}$ & $\begin{array}{l}26.8 \text { vs. } \\
45.3 \\
(\mathbf{p}<\mathbf{0 . 0 0 1 )}\end{array}$ & $\begin{array}{l}10 \text { vs. } 22.1 \\
(\mathbf{p}<\mathbf{0 . 0 0 1})\end{array}$ & & & $\begin{array}{l}59.1 \text { vs. } \\
45.4 \\
(\mathbf{p}<\mathbf{0 . 0 0 1})\end{array}$ & & $\begin{array}{l}\text { Anticardiolipin } \\
49.1 \text { vs. } 41.7(\mathbf{p}=\mathbf{0 . 0 3}) \\
\text { Anti-U1 nRNP } \\
28 \text { vs. } 13.3(\mathbf{p}<\mathbf{0 . 0 0 1}) \\
\text { Anti-Sm } \\
17.3 \text { vs. } 9.7(\mathbf{p}=\mathbf{0 . 0 0 4})\end{array}$ & & & $\begin{array}{l}21.9 \text { vs. } 17.8 \\
(p=0.14)\end{array}$ \\
\hline \multicolumn{11}{|c|}{ RA vs. RA-sSS patients } \\
\hline $\begin{array}{l}\text { Antero et al, } \\
2011\end{array}$ & & & $\begin{array}{l}58 \text { vs. } 70 \\
(p=0.24)\end{array}$ & $\begin{array}{l}30.6 \text { vs. } 30 \\
(p=0.95)\end{array}$ & & $\begin{array}{l}70.3 \text { vs. } \\
75 \\
(p=1.0)\end{array}$ & & N/A & & \\
\hline $\begin{array}{l}\text { Haga et al, } \\
2012\end{array}$ & & & $\begin{array}{l}156.46 \text { vs. } \\
54.90 \\
(p=N S)\end{array}$ & & & $\begin{array}{l}136.89 \\
\mathrm{U} / \mathrm{mL} \text { vs. } \\
125.17 \\
\mathrm{U} / \mathrm{mL} \\
(\mathrm{p}=\mathrm{NS})\end{array}$ & & $\begin{array}{l}\text { ESR } \\
20.53 \text { vs. } \\
14.90(p=N S) \\
\text { CRP } \\
1.52 \text { vs. } 1.20 \\
(p=N S)\end{array}$ & & \\
\hline $\begin{array}{l}\text { He J et al, } \\
2013\end{array}$ & $\begin{array}{l}4.69 \text { vs. } \\
39.2 \\
(\mathbf{p}=\mathbf{0 . 0 0 1 )}\end{array}$ & $\begin{array}{l}1.39 \text { vs. } \\
14.9 \\
(\mathbf{p}=\mathbf{0 . 0 0 1})\end{array}$ & $\begin{array}{l}75.6 \text { vs. } \\
95.7 \\
(p=0.001)\end{array}$ & $\begin{array}{l}51.7 \text { vs. } \\
79.8 \\
(\mathbf{p}=\mathbf{0 . 0 0 1})\end{array}$ & & $\begin{array}{l}71.9 \text { vs. } \\
77.8 \\
(\mathrm{p}=0.5)\end{array}$ & & $\mathrm{N} / \mathrm{A}$ & $\begin{array}{l}\text { IgA } \\
33.6 \text { vs. } 48.5 \\
(\mathbf{p}=\mathbf{0 . 1 0 6}) \\
\text { IgG } \\
35.6 \text { vs. } 54.3 \\
(\mathbf{p}=\mathbf{0 . 0 1 0}) \\
\text { IgM } \\
10.6 \text { vs. } 11.8 \\
(p=0.951)\end{array}$ & $\begin{array}{l}0.5 \text { vs. } 9.5 \\
(\mathbf{p}=\mathbf{0 . 0 0 1})\end{array}$ \\
\hline $\begin{array}{l}\text { Abdelghani } \\
\text { et al, } 2014\end{array}$ & $\begin{array}{l}1.5 \text { vs. } 0 \\
(p=1)\end{array}$ & & $\begin{array}{l}81.8 \text { vs. } \\
67.1 \\
(p=0.5)\end{array}$ & $\begin{array}{l}53.8 \text { vs. } \\
63.6(p=0.1)\end{array}$ & & $\begin{array}{l}64 \text { vs. } 90 \\
(p=0.1)\end{array}$ & $\begin{array}{l}\text { B2-m serum mean level } \\
1.9 \mathrm{mg} / \mathrm{l} \mathrm{vs.} 2.4 \\
\mathrm{mg} / \mathrm{l}(\mathrm{p}=0.02)\end{array}$ & $\begin{array}{l}\text { ESR } \\
20.9 \text { vs. } 24.8 \\
(p=0.4) \\
\text { CRP } \\
14 \text { vs. } 12.6\end{array}$ & & \\
\hline
\end{tabular}




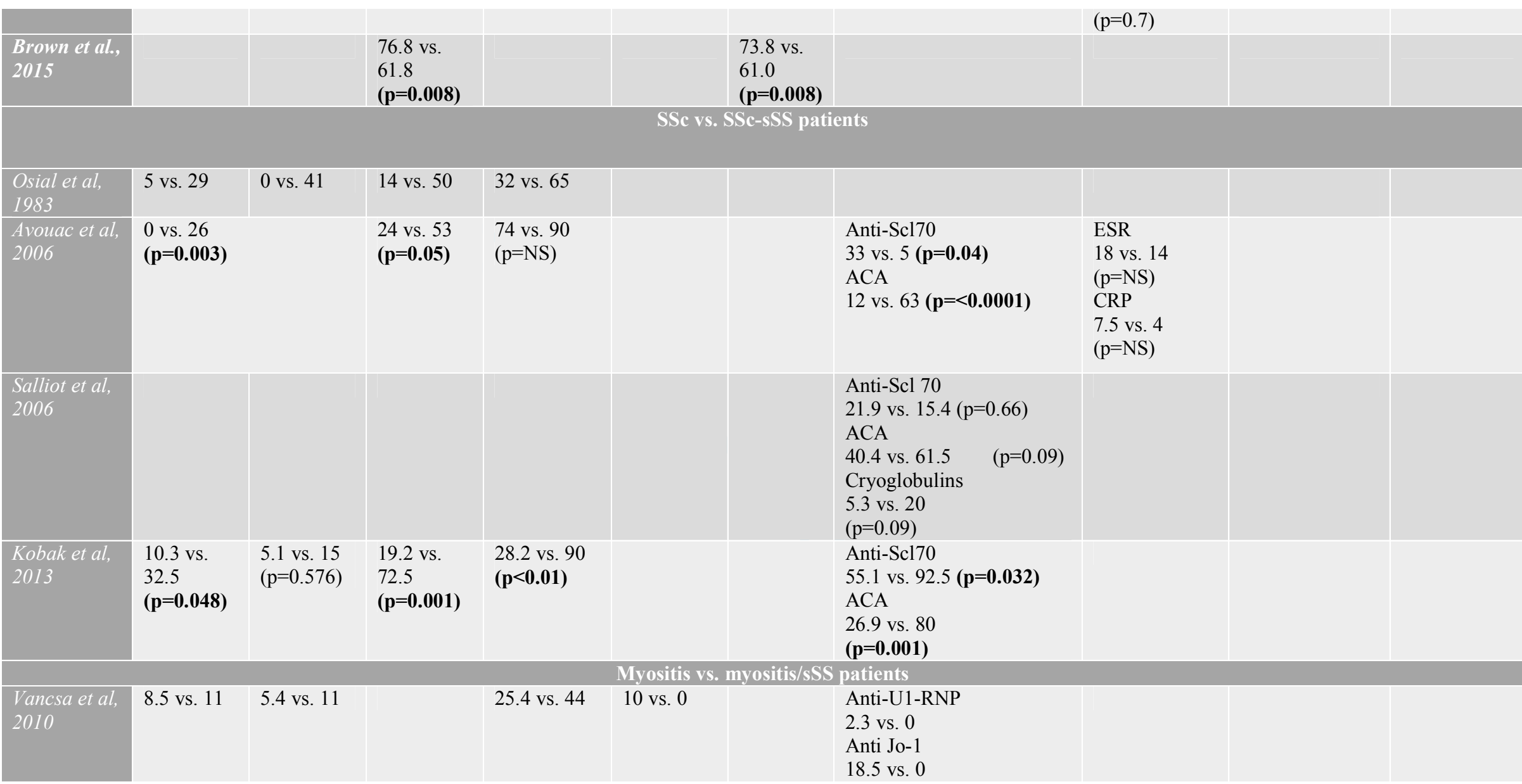

Legend: ACA- anti-centromere antibodies; anti-CCP- anti-cyclic citrullinated peptide antibody; anti-dsDNA - anti-double stranded DNA; ANA- antinuclear antibody; antiScl70 - anti-topoisomerase antibody; anti-Sm - anti-smith antibody; anti-U1-RNP - anti-nuclear ribonucleoprotein antibody; B2-m - beta 2 microglobulin; RF-Rheumatoid factor. 
Figure 1: Flowchart of study selection

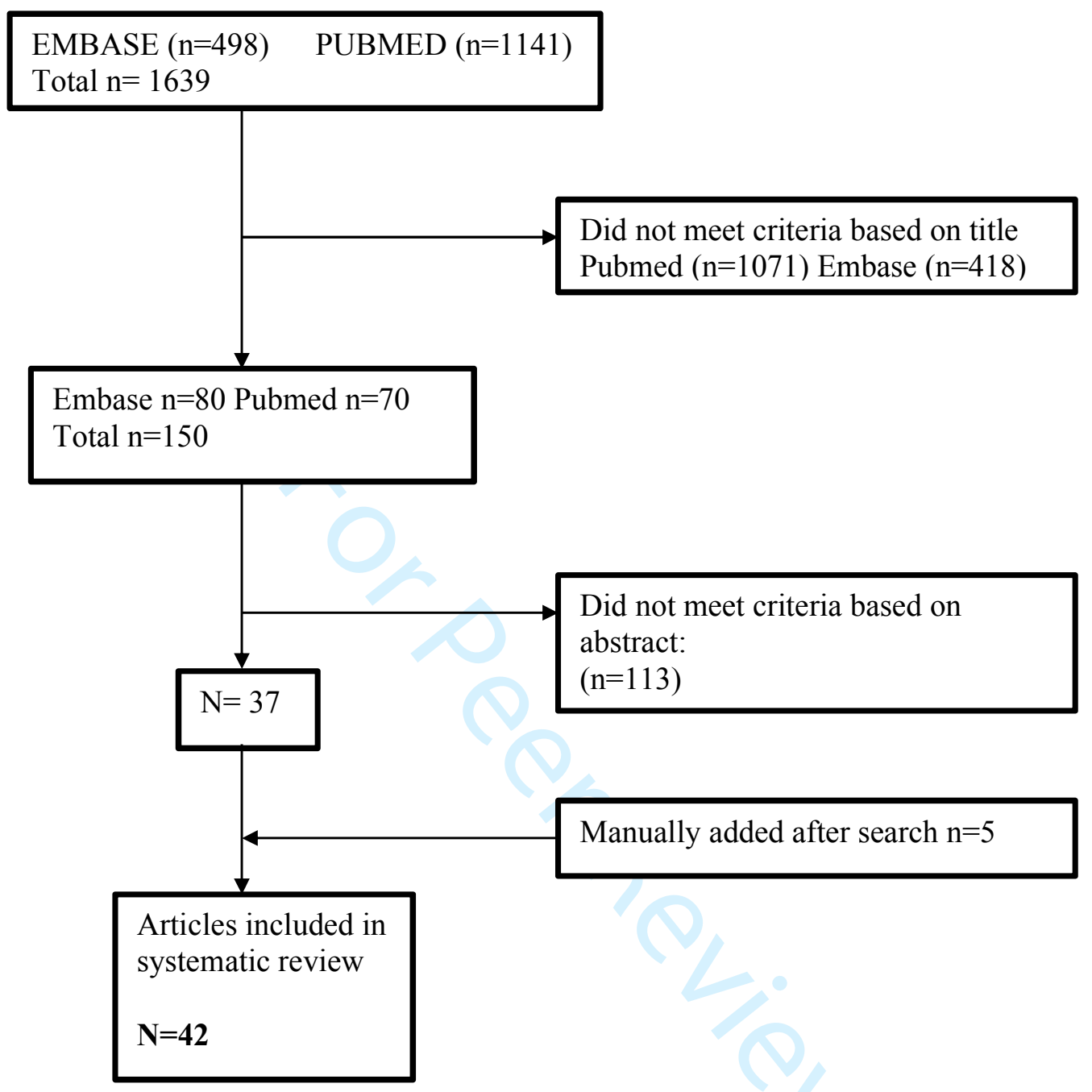


Bowman et al.,2003

McDonagh et al., 2000

Manoussakis et al., 2004

Gilboe et al., 2001

Pan et al., 2008

Aggarwal. et al., 2005

Szanto et al., 2006

Baer et al., 2000

Nossent et al., 1998

Pooled prevalence

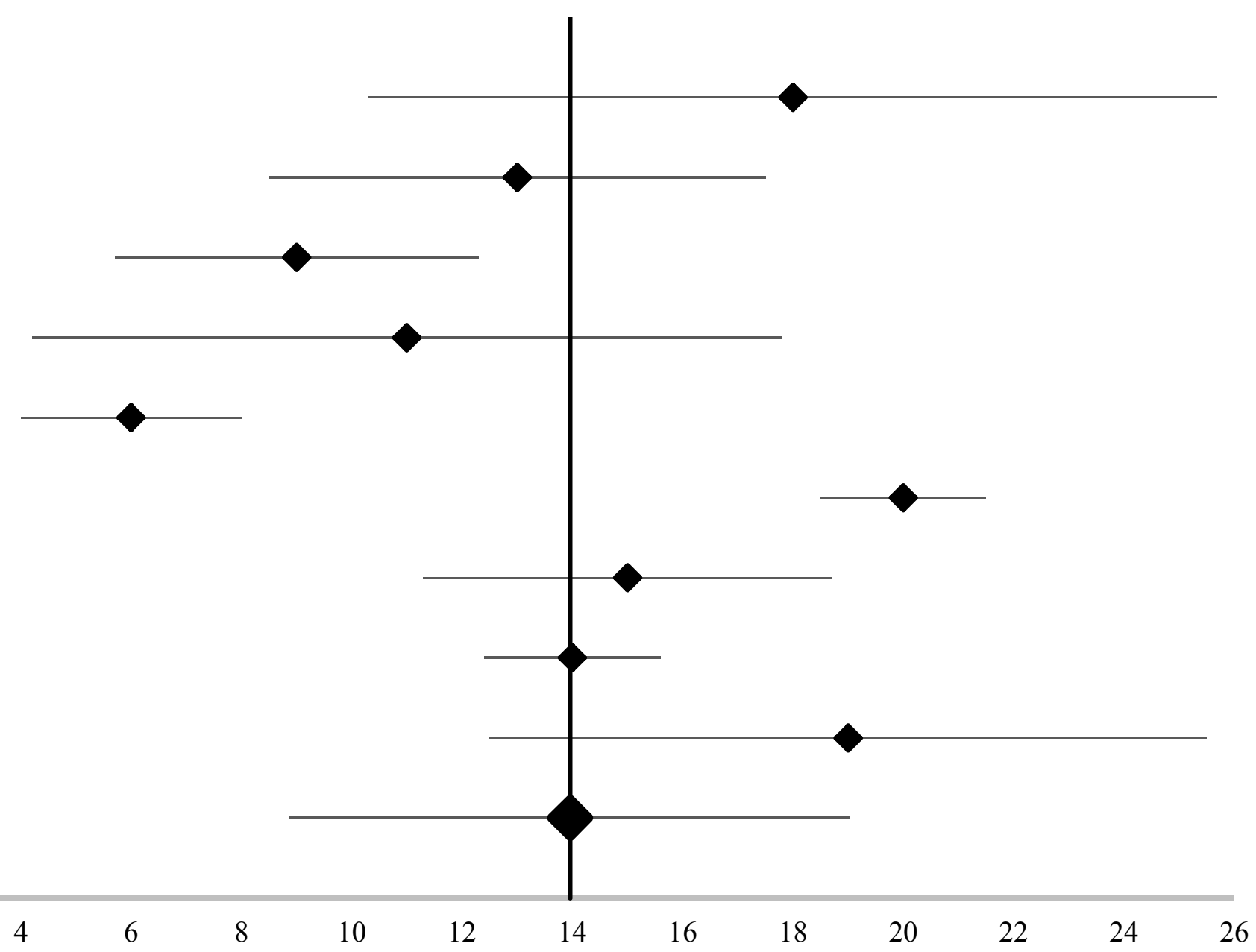


Figure 2: Meta-analysis of the prevalence of sSS in SLE patients.

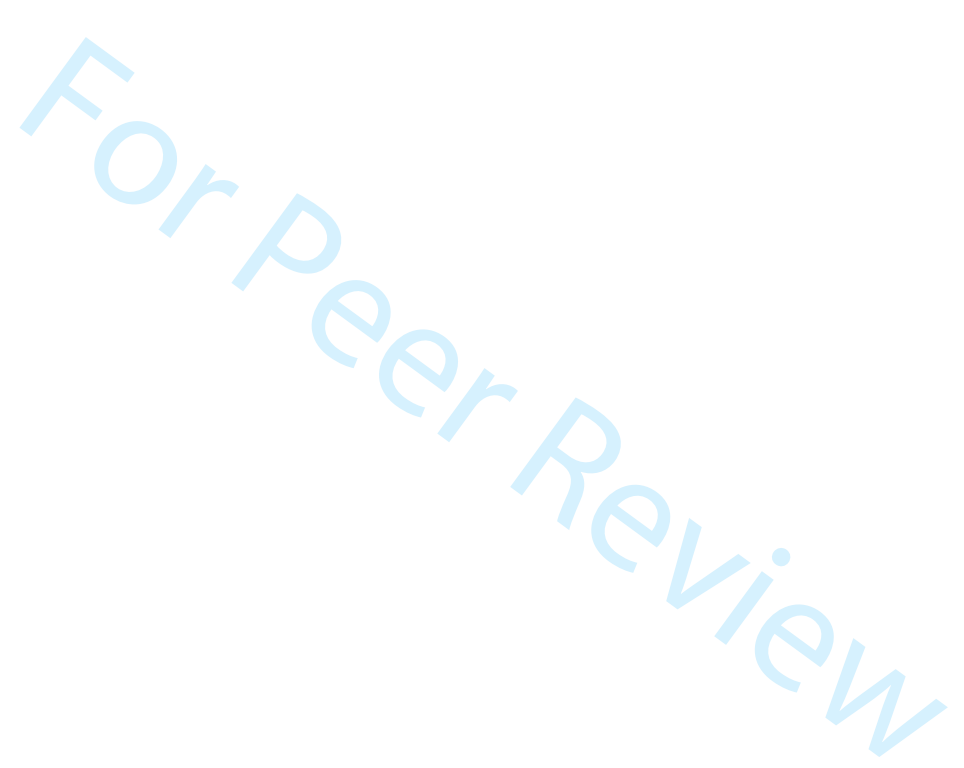




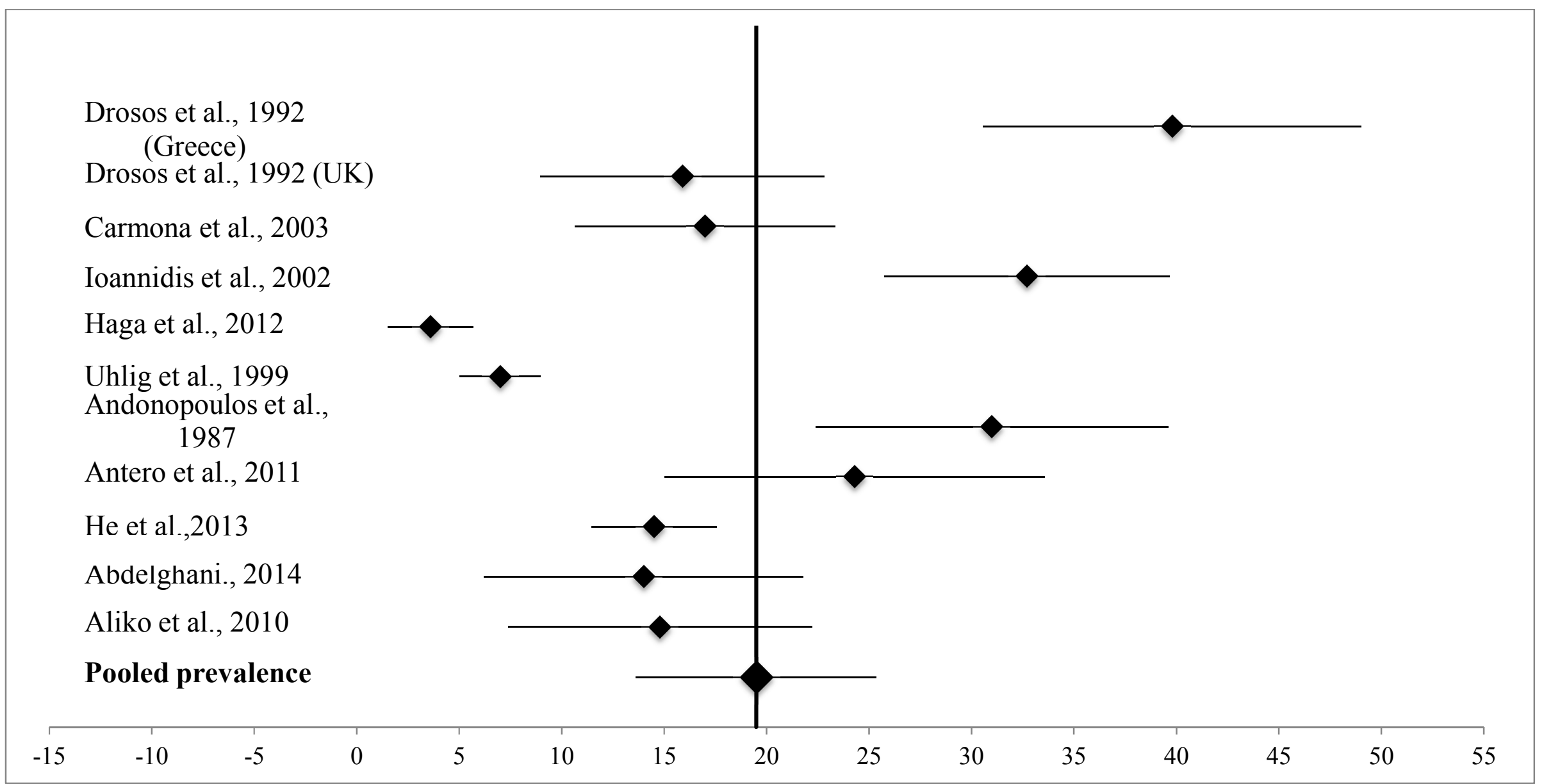

Figure 3: Meta-analysis of the prevalence of sSS in RA patients. 


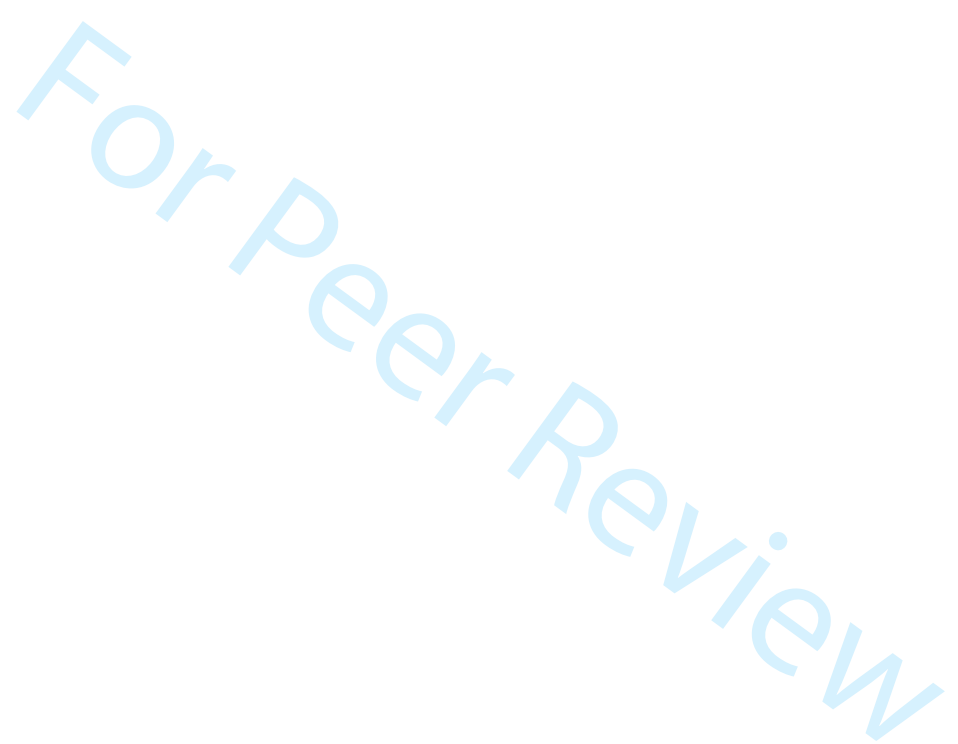

Scandinavian Journal of Rheumatology 